\title{
Green solution to corrosion problems - at a glance
}

\section{S. Rajendran, ${ }^{1} *$ R. Srinivasan, ${ }^{2}$ R. Dorothy, ${ }^{3}$ T. Umasankareswari ${ }^{4} *$ and A. Al-Hashem}

${ }^{1}$ Corrosion Research Centre, St Antony's College of Arts and Sciences for Women, Dindigul-624 005, India

${ }^{2}$ Member Secretary, Tamilnadu State Council for Science and Technology, Chennai, India

${ }^{3}$ AMET University, 135, Kanathur, East Coast Road, Chennai, India

${ }^{4}$ Department of Chemistry, Rajapalayam Rajus College, Rajapalayam, India

${ }^{5}$ Senior Scientist, Petroleum Research Center, Kuwait Institute for Scientific Research, Kuwait

*E-mail: susairajendran@gmail.com,venkatuma91@gmail.com

\begin{abstract}
Extracts of natural products are used as corrosion inhibitors since they are non toxic and environmentally friendly. Especially the extracts of leaves have attracted the attention of many researchers. Usually weight loss methods are adopted to measure the corrosion inhibition efficiencies. The mechanistic aspects have been investigated by electrochemical studies such as polarization study and $\mathrm{AC}$ impedance spectra. Sometimes noise measurement has also been used. The protective films formed on the metal surface have been analysed by surface analysis techniques such as SEM, EDAX, FTIR and AFM. Experiments have been carried out at various temperatures also. Various adsorption isotherms have been proposed. Of all the metals and alloys used, mild steel has been extensively used. The experiments have been carried out in acid medium and neutral medium (sodium chloride). The extracts of the leaves contain many ingredients which have polar groups such as $\mathrm{N}, \mathrm{O}, \mathrm{S}$ and $\mathrm{P}$ atoms. The lone pair of electrons present on these atoms are pumped on to the metal surface. This controls the corrosion process.
\end{abstract}

Key words: green inhibitors, corrosion inhibition, electrochemical studies, isotherms, SEM, AFM.

Received: February 3, 2019. Published: May 30, 2019

doi: $\underline{10.17675 / 2305-6894-2019-8-3-1}$

\section{Introduction}

When materials come in contact with environment, they decay. This decaying process is termed as corrosion. Even though most of the materials such as ceramics and glass also decay, usually, the term corrosion is concerned with metals. Corrosion is the expression of desire of the metals to go back to their original state of ores. Corrosion is a natural process, spontaneous and thermodynamically stable process favoured by nature. So no one can prevent corrosion. However the rate of corrosion can be controlled, just like postponing death. There are many methods to control corrosion. Anyhow the use of inhibitors in small 
quantities is a common process. Once chromates were used as corrosion inhibitors effectively. Yet, environmental scientists point out the health hazards caused by chromium(VI) ions. Hence corrosion scientists go for environmentally friendly non-toxic natural products extracts as corrosion inhibitors.

Many active principles are present in the extracts of plant materials. Usually they contain electron rich polar atoms such as oxygen, nitrogen, sulphur and phosphorus. These lone pair of electrons is pumped on to the metal surfaces. This prevents the metallic corrosion, because, loss of electrons by metals is prevented, by the pumped electrons. The recent trends in the use of green inhibitors are presented in this section. Many parts of the plant materials such as, flowers, barks, seeds and leaves can be used as corrosion inhibitors. The use of extracts of leaves as corrosion inhibitors is discussed. Several research papers have been published in this regard [1-104].

\section{Methods}

For evaluation of corrosion inhibition efficiencies of the extracts, methods such as weight loss methods [21, 22, 28, 31, 33, 35, 41, 42, 90] have been employed.

\section{Metals}

The corrosion of various metals such as mild steel $[2,21,22,25,26,27,28,31,32$, $35,36,40,41,42,47,53,90,92]$, stainless steel $[24,28,29,81]$, aluminium [3, 38, 44, 87], copper [33, 59], zinc [37], steel-copper [33], pipeline steel API 5L X52 [34], AM60 magnesium alloy [100] has been controlled by the extracts of leaves.

\section{Media}

The inhibition efficiencies of metals in various media such as acidic [2, 3, 21, $22,26,27,29,31,32,34,35,36,37,38,39,42,44,47,53,81,87,90,92]$, basic [88], neutral $[25,40,41,59,100]$ and sea water [103] have been investigated.

\section{Leaves}

Leaves such as Urtica dioica leaves extract [2], almond (Prunus amygdalus) fruit leaves [3], Xanthium strumarium leaves [21], Piper guineense (uziza leaf) [22], Olive leaves [24], Glycyrrhiza glabra [26], extract of ginkgo biloba [28], extract of Prunus persica ( $P$. persica) [29], Murraya koenigii (curry) leaves [32], Ziziyphus leaves [33], Aloe vera L. [35, 81], Gongronema latifolium [36], Areca palm leaves [38], Chaenomeles sinensis $(C$. sinensis) leaves [39], Ficus carica (Fig tree) leaves [40], Mangrove (Rhizophora apiculata) bark and leaf [42], Urtica Dioica leaves [43], Murraya koenigii leaves [44], Bauhinia tomentosa leaves extract [47], Schreabera swietenioids leaves [53], camellia sinensis [87], leaves of Pistacia lentiscus from Saidia Morocco [90], leaves of Hibiscus sabdariffa [92], have been used as corrosion inhibitors. 


\section{Mechanism of corrosion inhibition}

Mechanism of corrosion inhibition has been established by electrochemical studies such as polarization study $[24,27,29,35,41,42,44,59,90], \quad \mathrm{AC}$ impedance spectra $[24,25,27,29,35,38,42,44,59,81,90]$ electrochemical noise method [81]. The extracts can act as anodic inhibitors [44], cathodic inhibitors [29] and mixed type of inhibitors $[29,34,42,53]$.

\section{Surface analysis}

The protective films formed on the metal surface have been analysed by FTIR [24, 25, 31, 47, 53] spectra, SEM [2, 24, 28, 31, 38, 47, 53, 59, 81], EDAX [25], X-ray [32] and AFM $[2,26,38,47]$.

\section{Temperature}

Experiments have been carried out at various temperatures $[3,44,47]$ also.

\section{Isotherms}

The active principles of the extracts are adsorbed on the metal surface. The adsorption obeys Langmuir isotherm [37, 42, 44, 93], Freundlich isotherm [37, 93], Temkin [37], Frumkin [93], Florry-Huggins [93].

The outcomes of the study are summarized in Tables $1-5$.

Table 1. The outcomes of the study

\begin{tabular}{|c|c|c|c|c|c|}
\hline No. & $\begin{array}{c}\text { Metal / } \\
\text { Medium }\end{array}$ & $\begin{array}{c}\text { Inhibitor / } \\
\text { Additives }\end{array}$ & Methods & Findings & Ref. \\
\hline 1 & $\begin{array}{l}\text { Mild steel / } \\
\text { acidic media }\end{array}$ & $\begin{array}{c}\text { Parsley } \\
\text { (Petroselinum } \\
\text { Sativum) extract } \\
\text { (PSL) }\end{array}$ & $\begin{array}{l}\text { Weight loss, } \\
\text { potentiodynamic } \\
\text { polarization, } \\
\text { impedance } \\
\text { spectroscopy and } \\
\text { SEM }\end{array}$ & $\begin{array}{l}\text { PSL acts as a mixed type } \\
\text { inhibitor. } 92.39 \% \text { inhibition } \\
\text { efficiency (IE) }\end{array}$ & 1 \\
\hline 2 & $\begin{array}{l}\text { Mild steel } \\
\text { corrosion in } \\
1 \mathrm{M} \mathrm{HCl} \\
\text { solution }\end{array}$ & $\begin{array}{l}\text { Urtica dioica } \\
\text { leaves extract }\end{array}$ & $\begin{array}{l}\text { EIS, polarization } \\
\text { test, SEM, AFM and } \\
\text { computational } \\
\text { approaches }\end{array}$ & $\begin{array}{l}\text { adsorption of green inhibitors } \\
\text { on the steel substrate, the } \\
\text { density functional theory } \\
\text { calculations suggested the } \\
\text { inhibitors adsorption via } \\
\text { electronic donor-acceptor } \\
\text { interactions. } 92 \% \text { IE }\end{array}$ & 2 \\
\hline 3 & $\begin{array}{c}\text { Aluminium } \\
\text { corrosion in } \\
\mathrm{HCl} \text { acidic } \\
\text { medium }\end{array}$ & $\begin{array}{l}\text { Almond (Prunus } \\
\text { amygdalus) fruit } \\
\text { leaves extract as } \\
\text { green inhibitor }\end{array}$ & $\begin{array}{l}\text { Optimisation and } \\
\text { modeling, effect of } \\
\text { temperature, } \\
\text { Response Surface }\end{array}$ & $97.9 \% \mathrm{IE}$ & 3 \\
\hline
\end{tabular}




\begin{tabular}{|c|c|c|c|c|c|}
\hline No. & $\begin{array}{l}\text { Metal / } \\
\text { Medium }\end{array}$ & $\begin{array}{l}\text { Inhibitor / } \\
\text { Additives }\end{array}$ & Methods & Findings & Ref. \\
\hline & & & Methodology (RSM) & & \\
\hline 4 & $\begin{array}{l}\text { Steel in } \\
\text { chloride } \\
\text { solutions }\end{array}$ & Olive leaf extract & Polarization, EIS & $\begin{array}{l}80 \% \text { IE for ethanol extracted } \\
\text { inhibitor at } 300 \mathrm{ppm}\end{array}$ & 4 \\
\hline 5 & $\begin{array}{l}\text { Aluminium } \\
\text { corrosion in } \\
1 \mathrm{M} \mathrm{HCl}\end{array}$ & $\begin{array}{l}\text { Orange seed } \\
\text { extract }\end{array}$ & $\begin{array}{c}\text { Weight loss } \\
\text { measurement and } \\
\text { phytochemical } \\
\text { analysis, temperature } \\
\text { study }\end{array}$ & $\begin{array}{c}38.37 \% \text { IE. Orange seed } \\
\text { extract is a good corrosion } \\
\text { inhibitor }\end{array}$ & 5 \\
\hline 6 & $\begin{array}{l}\text { Steel } \\
\text { corrosion }\end{array}$ & $\begin{array}{c}\text { Silver } \\
\text { nanoparticles } \\
\text { (AgNPs) doped } \\
\text { palm oil leaf }\end{array}$ & $\begin{array}{l}\text { Linear polarization } \\
\text { resistance (LPR), } \\
\text { potentiodynamic } \\
\text { polarisation, half- } \\
\text { cell potential (HCP) } \\
\text { and electrical } \\
\text { resistivity }\end{array}$ & $\begin{array}{l}\text { Maximum inhibition efficiency } \\
\text { was recorded to be as much as } \\
94.74 \%\end{array}$ & 6 \\
\hline 7 & $\begin{array}{l}\text { Benzo- } \\
\text { triazole } \\
\text { uptake and } \\
\text { removal in } \\
\text { vegetated } \\
\text { biofilter } \\
\text { mesocosms } \\
\text { planted with } \\
\text { Carex } \\
\text { praegracilis }\end{array}$ & $\begin{array}{l}\text { 1H-Benzotriazole } \\
\text { is a persistent, } \\
\text { recalcitrant trace } \\
\text { organic } \\
\text { contaminant } \\
\text { commonly used } \\
\text { as a corrosion } \\
\text { inhibitor in } \\
\text { airplane deicing } \\
\text { processes, } \\
\text { automobile } \\
\text { liquids, and } \\
\text { engine coolants. }\end{array}$ & $\begin{array}{l}\text { Removal of } 1 H \text { - } \\
\text { benzotriazole from } \\
\text { stormwater using } \\
\text { bench-scale biofilter } \\
\text { mesocosms planted } \\
\text { with California } \\
\text { native sedge, Carex } \\
\text { praegracilis, over a } \\
\text { series of three storm } \\
\text { events and } \\
\text { succeeding } \\
\text { monitoring period. }\end{array}$ & $\begin{array}{l}\text { This study suggests that } \\
\text { vegetation may increase the } \\
\text { operating lifespan of } \\
\text { bioretention basins by } \\
\text { enhancing the degradation of } \\
\text { dissolved trace organic } \\
\text { contaminants, thus increasing } \\
\text { the sorption capacity of the } \\
\text { geomedia. }\end{array}$ & \\
\hline 8 & Mild steel & $\begin{array}{c}\text { Zinc } \\
\text { acetylacetonate }\end{array}$ & $\begin{array}{c}\text { Scanning electron } \\
\text { microscopy (SEM), } \\
\text { Fourier transform } \\
\text { infrared } \\
\text { spectroscopy (FT- } \\
\text { IR), UV-Vis } \\
\text { analysis, thermal } \\
\text { gravimetric analysis } \\
\text { (TGA), and X-ray } \\
\text { photoelectron } \\
\text { spectroscopy (XPS) } \\
\text { techniques }\end{array}$ & $\begin{array}{l}\text { Pigment showed mixed } \\
\text { corrosion inhibition properties. }\end{array}$ & 7 \\
\hline 9 & Mild steel in & Elaeis guineensis & Weight loss & EG leaves is a potential metal & 8 \\
\hline
\end{tabular}




\begin{tabular}{|c|c|c|c|c|c|}
\hline No. & $\begin{array}{l}\text { Metal / } \\
\text { Medium }\end{array}$ & $\begin{array}{l}\text { Inhibitor / } \\
\text { Additives }\end{array}$ & Methods & Findings & Ref. \\
\hline & $\begin{array}{l}\text { hydrochloric } \\
\text { acid }\end{array}$ & leaves extracts & $\begin{array}{c}\text { measurement, } \\
\text { Adsorption, SEM } \\
\text { and EDX analyses }\end{array}$ & $\begin{array}{l}\text { corrosion inhibitor in acidic } \\
\text { condition }\end{array}$ & \\
\hline 10 & $\begin{array}{l}\text { Mild steel in } \\
\text { sea water }\end{array}$ & $\begin{array}{l}\text { Azadirachta } \\
\text { indica L. extracts }\end{array}$ & $\begin{array}{c}\text { Weight loss, } \\
\text { electrochemical } \\
\text { impedance } \\
\text { spectroscopy (EIS), } \\
\text { linear polarization } \\
\text { and potentiodynamic } \\
\text { polarization } \\
\text { techniques }\end{array}$ & $98 \% \mathrm{IE}$ & 9 \\
\hline 11 & $\begin{array}{l}\text { E } 24 \text { steel in } \\
\text { a neutral } \\
\text { solution } \\
\mathrm{NaCl} 3.5 \%\end{array}$ & $\begin{array}{c}\text { Methanolic } \\
\text { extract }\end{array}$ & $\begin{array}{l}\text { Scanning electron } \\
\text { microscope (SEM) } \\
\text { coupled with the } \\
\text { EDS }\end{array}$ & Inhibitors act as a mixed type & 10 \\
\hline 12 & $\begin{array}{l}\text { Austenitic } \\
\text { stainless } \\
\text { steel }(304 S S \\
\text { corrosion in } \\
\text { conc. } \mathrm{HCl})\end{array}$ & $\begin{array}{l}\text { Psidium guajava } \\
\text { leaves }\end{array}$ & $\begin{array}{l}\text { Polarization } \\
\text { potentiodynamic } \\
\text { measurements }\end{array}$ & $\begin{array}{l}\text { Inhibition efficiency up to } \\
91.81 \% \text {. }\end{array}$ & 11 \\
\hline 13 & $\begin{array}{l}\text { Metals and } \\
\text { alloys in } \\
\text { aggressive } \\
\text { corrosive } \\
\text { media }\end{array}$ & $\begin{array}{l}\text { Green inhibition } \\
\text { strategies }\end{array}$ & - & $\begin{array}{l}\text { Plant extract as corrosion } \\
\text { inhibitors for metals and alloys } \\
\text { in aggressive aqueous solutions }\end{array}$ & 12 \\
\hline 14 & $\begin{array}{l}\mathrm{Cu} \text { in Nitric } \\
\text { acid } \\
\text { solutions }\end{array}$ & Berry leaves & $\begin{array}{c}\text { Electrochemical } \\
\text { impedance } \\
\text { spectroscopy (EIS), } \\
\text { potentiodynamic } \\
\text { polarization (PP), } \\
\text { electrochemical } \\
\text { frequency } \\
\text { modulation (EFM), } \\
\text { and weight loss } \\
\text { techniques }\end{array}$ & Mixed-type inhibitor & \\
\hline 15 & $\begin{array}{l}\text { Mild steel in } \\
1 \mathrm{M} \\
\text { hydrochloric } \\
\text { acid }\end{array}$ & $\begin{array}{l}\text { Holoptelea } \\
\text { integrifolia leaf } \\
\text { extract (HILE) }\end{array}$ & $\begin{array}{l}\text { weight loss, surface, } \\
\text { electrochemical, and } \\
\text { DFT methods, SEM } \\
\text { and AFM }\end{array}$ & $93.91 \%$ IE & 14 \\
\hline
\end{tabular}




\begin{tabular}{|c|c|c|c|c|c|}
\hline No. & $\begin{array}{l}\text { Metal / } \\
\text { Medium }\end{array}$ & $\begin{array}{l}\text { Inhibitor / } \\
\text { Additives }\end{array}$ & Methods & Findings & Ref. \\
\hline 16 & $\begin{array}{l}304 \mathrm{~L} \\
\text { stainless } \\
\text { steel }\end{array}$ & $\begin{array}{l}\text { Cistus ladanifer } \\
\text { leaves extract }\end{array}$ & $\begin{array}{l}\text { Electrochemical } \\
\text { measurements and } \\
\text { surface analysis } \\
\text { techniques }\end{array}$ & $99.36 \%$ IE & 15 \\
\hline 17 & $\begin{array}{l}\text { Mild steel in } \\
1 \mathrm{~N} \text { sulfuric } \\
\text { acid } \\
\text { medium }\end{array}$ & $\begin{array}{l}\text { Pongamia } \\
\text { Pinnata }\end{array}$ & $\begin{array}{l}\text { Weight loss method, } \\
\text { potentiodynamic } \\
\text { polarization method } \\
\text { and electrochemical } \\
\text { impedance } \\
\text { spectroscopy (EIS) } \\
\text { technique, Fourier } \\
\text { transform infrared } \\
\text { spectroscopy (FTIR) } \\
\text { and gas } \\
\text { chromatography- } \\
\text { mass spectrometry } \\
\text { (GCMS) analysis }\end{array}$ & $\begin{array}{c}\text { Maximum inhibition efficiency } \\
\text { was attained at } 100 \mathrm{ppm} \text { of the } \\
\text { leaf extract }\end{array}$ & 16 \\
\hline 18 & $\begin{array}{c}\text { Steel } \\
\text { dissolution } \\
\text { in acidic } \\
\text { medium }\end{array}$ & $\begin{array}{l}\text { Glycine max, } \\
\text { Cuscuta reflexa } \\
\text { and Spirogyra } \\
\text { extracts }\end{array}$ & $\begin{array}{l}\text { Electrochemical, } \\
\text { surface and density } \\
\text { functional theory } \\
\text { (DFT) methods }\end{array}$ & $\begin{array}{l}\text { IE: GMLE > CRRE > SGAE. } \\
73.60 \%, 81.92 \% \text { and } 94.05 \%\end{array}$ & 17 \\
\hline 19 & $\begin{array}{l}\text { Mild steel } \\
\text { against acid } \\
\text { activation }\end{array}$ & $\begin{array}{l}\text { Novel Elaeis } \\
\text { Guineensis and } \\
\text { silver } \\
\text { nanoparticles }\end{array}$ & $\begin{array}{c}\text { FESEM, EDX, } \\
\text { AFM, XRD, weight } \\
\text { loss, polarization and } \\
\text { electrochemical } \\
\text { impedance } \\
\text { measurements , } \\
\text { TEM, XRD, and } \\
\text { EDX analyses }\end{array}$ & $94.1 \% \mathrm{IE}$ & 18 \\
\hline 20 & $\begin{array}{l}316 \mathrm{~L} \\
\text { stainless } \\
\text { steel }\end{array}$ & $\begin{array}{l}\text { Green inhibitors } \\
\text { (leaf extracts of } \\
\text { Musa spp. (MS) } \\
\text { and Jatropha } \\
\text { curcas (JC)) }\end{array}$ & $\begin{array}{l}\text { Current transients } \\
\text { and potentiodynamic } \\
\text { polarization }\end{array}$ & Cathodic inhibitor & 19 \\
\hline
\end{tabular}

Table 2. Outcomes of the study

\begin{tabular}{|c|c|c|c|c|c|}
\hline No. & $\begin{array}{c}\text { Metal / } \\
\text { Medium }\end{array}$ & $\begin{array}{c}\text { Inhibitor / } \\
\text { Additives }\end{array}$ & Methods & Findings & Ref. \\
\hline 21 & $\begin{array}{c}\text { Low carbon } \\
\text { steel in HCl }\end{array}$ & $\begin{array}{c}\text { Xanthium } \\
\text { strumarium } \\
\text { leaves }\end{array}$ & Weight loss method & $\begin{array}{c}\text { The inhibition efficiency was } \\
\text { found to increases with } \\
\text { increase in inhibitor }\end{array}$ & 21 \\
\hline
\end{tabular}




\begin{tabular}{|c|c|c|c|c|c|}
\hline No. & $\begin{array}{c}\text { Metal / } \\
\text { Medium }\end{array}$ & $\begin{array}{l}\text { Inhibitor / } \\
\text { Additives }\end{array}$ & Methods & Findings & Ref. \\
\hline & & & & concentration and temperature & \\
\hline 22 & $\begin{array}{l}\text { Mild steel in } \\
\text { a } 2 \mathrm{M} \mathrm{H}_{2} \mathrm{SO}_{4} \\
\text { medium }\end{array}$ & $\begin{array}{l}\text { Piper guineense } \\
\text { (uziza leaf) } \\
\text { extract }\end{array}$ & Weight loss method & $\begin{array}{l}\text { Corrosion inhibition increases } \\
\text { with increasing concentrations } \\
\text { of Piper guineense extract }\end{array}$ & 22 \\
\hline 23 & $\begin{array}{l}\text { Metals when } \\
\text { exposed to } \\
\text { acidic } \\
\text { environment }\end{array}$ & $\begin{array}{l}\text { Synthetic } \\
\text { polymers }\end{array}$ & & & 23 \\
\hline 24 & $\begin{array}{l}\text { Stainless } \\
\text { steel grade } \\
\text { 316L } \\
(\mathrm{SS} 316 \mathrm{~L})\end{array}$ & $\begin{array}{l}\text { Olive leaves } \\
\text { extract (OLE) }\end{array}$ & $\begin{array}{l}\text { Scanning electron } \\
\text { microscope (SEM), } \\
\text { electrochemical } \\
\text { impedance } \\
\text { spectroscopy (EIS) } \\
\text { and potentiodynamic } \\
\text { polarization } \\
\text { techniques. The } \\
\text { Fourier Transform } \\
\text { Infrared (FTIR) } \\
\text { spectroscopy }\end{array}$ & $\begin{array}{l}\text { OLE as anticorrosion coating } \\
\text { for control of stainless steel in } \\
\text { marine application. }\end{array}$ & 24 \\
\hline 25 & $\begin{array}{l}\text { Mild steel } \\
\text { corrosion in } \\
3.5 \% \mathrm{NaCl} \\
\text { solution }\end{array}$ & $\begin{array}{l}\text { Green Nettle } \\
\text { leaves extract }\end{array}$ & $\begin{array}{l}\text { Molecular dynamics } \\
\text { (MD) simulations } \\
\text { and quantum } \\
\text { mechanics (QM) } \\
\text { methods, (EIS), (FT- } \\
\text { IR), (EDS) }\end{array}$ & $\begin{array}{l}\text { MD simulations demonstrated } \\
\text { the adsorption ability of } \\
\text { organic-inorganic inhibitors } \\
\text { onto steel substrate. }\end{array}$ & 25 \\
\hline 26 & $\begin{array}{l}\text { Mild steel } \\
1 \mathrm{M} \mathrm{HCl} \\
\text { solution }\end{array}$ & $\begin{array}{l}\text { Glycyrrhiza } \\
\text { glabra extract }\end{array}$ & $\begin{array}{l}\text { Atomic force } \\
\text { microscopy test }\end{array}$ & $\begin{array}{c}\text { All corrosion } \\
\text { inhibiting materials exist in } \\
\text { Glycyrrhiza glabra adsorbed to } \\
\text { steel surface, and thereby form } \\
\text { a corrosion-protective film } \\
\text { over the steel surface. }\end{array}$ & 26 \\
\hline 27 & $\begin{array}{l}\text { Mild steel in } \\
0.5 \mathrm{M} \mathrm{H}_{2} \mathrm{SO}_{4}\end{array}$ & $\begin{array}{l}\text { Dried marjoram } \\
\text { leaves (DML) }\end{array}$ & $\begin{array}{l}\text { Anodic and cathodic } \\
\text { polarization and } \\
\text { electrochemical } \\
\text { impedance } \\
\text { spectroscopy (EIS) } \\
\text { measurements } \\
\end{array}$ & $\begin{array}{l}\text { The protection efficiency } \\
\text { increases with increasing } \\
\text { concentration of DML and } \\
\text { decreasing temperatures. }\end{array}$ & 27 \\
\hline 28 & $\begin{array}{l}\mathrm{N} 80 \text { steel in } \\
5 \% \mathrm{HCl} \\
\text { solution }\end{array}$ & $\begin{array}{c}\text { Extract of ginkgo } \\
\text { biloba }\end{array}$ & $\begin{array}{l}\text { (SEM) and weight } \\
\text { loss }\end{array}$ & $\begin{array}{l}\text { The surface topography of the } \\
\text { steels before and after being } \\
\text { corrupted showed that the } \\
\text { extracts offer good protection }\end{array}$ & 28 \\
\hline
\end{tabular}




\begin{tabular}{|c|c|c|c|c|c|}
\hline No. & $\begin{array}{l}\text { Metal / } \\
\text { Medium }\end{array}$ & $\begin{array}{l}\text { Inhibitor / } \\
\text { Additives }\end{array}$ & Methods & Findings & Ref. \\
\hline 29 & $\begin{array}{l}\text { AISI } 1018 \\
\text { steel in } \\
0.5 \mathrm{M} \mathrm{H}_{2} \mathrm{SO}_{4} \\
\text { at } 25^{\circ} \mathrm{C} \text {. }\end{array}$ & $\begin{array}{l}\text { Extract of Prunus } \\
\text { persica }(P . \\
\text { persica }) \text { leaves }\end{array}$ & (CIE), (PPC), (EIS) & $\begin{array}{l}\text { The analysis of the } \\
\text { electrochemical parameters } \\
\text { from the polarizations curves } \\
\text { confirmed that the } P \text {. persica } \\
\text { extract behaved as a mixed- } \\
\text { type GCI with cathodic } \\
\text { predominance. }\end{array}$ & 29 \\
\hline 30 & $\begin{array}{l}\text { Antimicrobial } \\
\text { actions }\end{array}$ & $\begin{array}{c}\text { Silver } \\
\text { nanoparticles (Ag- } \\
\text { NPs) using Sida } \\
\text { acuta extract }\end{array}$ & $\begin{array}{l}\text { X-ray diffraction and } \\
\text { transmission electron } \\
\text { microscopy (TEM) }\end{array}$ & $\begin{array}{l}\text { The infrared spectra confirmed } \\
\text { the bio-fabrication of the Ag- } \\
\text { NPs displayed the existence of } \\
\text { conceivable functional groups } \\
\text { responsible for the bio- } \\
\text { reduction and capping. }\end{array}$ & 30 \\
\hline 31 & $\begin{array}{l}\text { Mild steel in } \\
0.1 \mathrm{M} \text { of } \\
\text { hydrochloric } \\
\text { acid }(\mathrm{HCl})\end{array}$ & $\begin{array}{l}\text { Black tea extract } \\
\text { containing } \\
\text { compounds such } \\
\text { as catechin, } \\
\text { caffeine and } \\
\text { tannins }\end{array}$ & $\begin{array}{l}\text { Corrosion weight } \\
\text { loss measurement, } \\
\text { FTIR, SEM }\end{array}$ & $\begin{array}{l}\text { Loss in weight of mild steel } \\
\text { reduces as the concentration of } \\
\text { inhibitor increases. }\end{array}$ & 31 \\
\hline 32 & $\begin{array}{l}\text { Mild steel } \\
(\mathrm{MS}) \text { in } \\
\text { nitric acid } \\
\text { medium }\end{array}$ & $\begin{array}{l}\text { Murraya koenigii } \\
\text { (curry) leaves } \\
\text { extract }\end{array}$ & $\begin{array}{l}\text { Adsorption isotherm } \\
\text { studies and the } \\
\text { thermodynamic } \\
\text { analysis, X-ray } \\
\text { spectroscopy }\end{array}$ & $\begin{array}{c}\text { Mild steel corrosion inhibition } \\
\text { efficiency of } 62 \% \text { is obtained } \\
\text { with } 600 \text { ppm of extract in } \\
0.1 \mathrm{~N} \mathrm{HNO}_{3} \text {. }\end{array}$ & 32 \\
\hline 33 & Steel-copper & $\begin{array}{c}\text { Ziziphus leaves } \\
\text { extract }\end{array}$ & $\begin{array}{l}\text { Weight loss } \\
\text { technique }\end{array}$ & $\begin{array}{l}\text { Inhibition efficiency increases } \\
\text { with increase in inhibitor } \\
\text { concentration but decrease with } \\
\text { rise in temperature. }\end{array}$ & 33 \\
\hline 34 & $\begin{array}{l}\text { Pipeline } \\
\text { steel API 5L } \\
\text { X52 in } \\
\text { hydrochloric } \\
\text { acid solution }\end{array}$ & $\begin{array}{l}\text { Extract from the } \\
\text { leaves of Ruta } \\
\text { Chalepensis } \\
\text { (LERC) }\end{array}$ & & $\begin{array}{l}\text { LERC inhibitor affects both the } \\
\text { anodic and cathodic corrosion } \\
\text { reactions. }\end{array}$ & 34 \\
\hline 35 & $\begin{array}{l}\text { Mild steel in } \\
5.0 \mathrm{M} \\
\text { hydrochloric } \\
\text { acid }\end{array}$ & $\begin{array}{c}\text { Aloe vera } L . \\
\text { extract }\end{array}$ & $\begin{array}{l}\text { Weight loss, } \\
\text { gasometric, } \\
\text { potentiodynamic } \\
\text { polarization and } \\
\text { electrochemical } \\
\text { impedance } \\
\text { spectroscopy (EIS) } \\
\text { techniques }\end{array}$ & $\begin{array}{l}97 \% \text { corrosion inhibition } \\
\text { efficiency was shown by } 4 \mathrm{~g} / \mathrm{L} \\
\text { of Aloe vera } L . \text { in } 5 \mathrm{M} \mathrm{HCl} \\
\text { solution for mild steel. }\end{array}$ & 35 \\
\hline
\end{tabular}




\begin{tabular}{|c|c|c|c|c|c|}
\hline No. & $\begin{array}{l}\text { Metal / } \\
\text { Medium }\end{array}$ & $\begin{array}{l}\text { Inhibitor / } \\
\text { Additives }\end{array}$ & Methods & Findings & Ref. \\
\hline 36 & $\begin{array}{l}\text { Mild steel in } \\
\text { acid media }\end{array}$ & $\begin{array}{l}\text { Extracted from } \\
\text { Gongronema } \\
\text { latifolium } \\
\text { (SEGL) } \\
\end{array}$ & $\begin{array}{l}\text { Hydrogen evolution } \\
\text { technique }\end{array}$ & $\begin{array}{l}\text { Inhibition efficiency depends } \\
\text { on the concentration of the } \\
\text { plant extract, temperature and } \\
\text { the period of immersion. }\end{array}$ & 36 \\
\hline 37 & $\begin{array}{c}\text { Zinc } \\
\text { corrosion in } \\
\text { acidic } \\
\text { mediated }\end{array}$ & $\begin{array}{l}\text { Neem and } \\
\text { Hibiscus, as a } \\
\text { green inhibitor }\end{array}$ & Analysis adsorption & $\begin{array}{l}\text { The Freundlich, Langmuir and } \\
\text { Temkin models are employed } \\
\text { to analysis adsorption occurred } \\
\text { in the experiment. }\end{array}$ & 37 \\
\hline 38 & $\begin{array}{l}\text { Aluminium } \\
\text { corrosion } \\
\text { inhibition in } \\
\text { hydrochloric } \\
\text { acid }(0.5 \mathrm{M}) \\
\text { medium }\end{array}$ & $\begin{array}{l}\text { Areca palm } \\
\text { leaves }(A L) \\
\text { extract }\end{array}$ & $\begin{array}{l}\text { Scanning electron } \\
\text { microscopy (SEM) } \\
\text { and atomic force } \\
\text { microscopy (AFM) } \\
\text { techniques }\end{array}$ & $\begin{array}{l}\text { Impedance method indicates } \\
\text { that the Al dissolution in } \\
\text { hydrochloric acid environment } \\
\text { was fully hindered by charge } \\
\text { transfer process. }\end{array}$ & 38 \\
\hline 39 & $\begin{array}{c}\text { Acid } \\
\text { corrosion of } \\
\text { low carbon } \\
\text { steel }\end{array}$ & $\begin{array}{l}\text { Chaenomeles } \\
\quad \text { sinensis } \\
\text { (C. sinensis) } \\
\quad \text { leaves }\end{array}$ & $\begin{array}{l}\text { Phytochemical } \\
\text { characterization }\end{array}$ & $\begin{array}{l}\text { Efficiency increased } \\
\text { remarkably in the presence of } \\
\text { inhibitor and found as } \\
\text { concentration dependent. }\end{array}$ & 39 \\
\hline 40 & $\begin{array}{l}\text { Mild steel } \\
3.5 \% \text { sodium } \\
\text { chloride } \\
\text { solution }\end{array}$ & $\begin{array}{c}\text { Ficus carica (Fig } \\
\text { tree) leaves } \\
\text { extract }\end{array}$ & $\begin{array}{l}\text { Electrochemical } \\
\text { techniques }\end{array}$ & $\begin{array}{l}\text { Among the four major Ficus } \\
\text { carica leaves extract } \\
\text { constituent investigated, } \\
\text { Caffeoylmalic acid was found } \\
\text { to make the most contribution } \\
\text { to the overall inhibition action } \\
\text { of Ficus carica leaves extract. }\end{array}$ & 40 \\
\hline
\end{tabular}

Table 3. Outcomes of the study

\begin{tabular}{|c|c|c|c|c|c|}
\hline No. & $\begin{array}{c}\text { Metal / } \\
\text { Medium }\end{array}$ & $\begin{array}{c}\text { Inhibitor / } \\
\text { Additives }\end{array}$ & Methods & Findings & Ref. \\
\hline 41 & $\begin{array}{c}\text { Carbon steel } \\
\text { in NaCl } \\
\text { solution }\end{array}$ & $\begin{array}{c}\text { Key lime (Citrus } \\
\text { Aurantiifolia) } \\
\text { leaves and seeds } \\
\text { at pH=7 }\end{array}$ & $\begin{array}{c}\text { Weight loss method, } \\
\text { polarisation study }\end{array}$ & Good inhibition efficiency. & 41 \\
\hline 2 & $\begin{array}{c}\text { Mild steel in } \\
1 \mathrm{M} \mathrm{HCl}\end{array}$ & $\begin{array}{c}\text { Mangrove } \\
\text { (Rhizophora } \\
\text { apiculata) bark } \\
\text { and leaf }\end{array}$ & $\begin{array}{c}\text { Gravimetric } \\
\text { (weight loss) } \\
\text { electrochemical } \\
\text { impedance } \\
\text { spectroscopy (EIS) } \\
\text { and potentiodynamic } \\
\text { polarization }\end{array}$ & $\begin{array}{c}\text { Mixed type inhibitor, } \\
\text { Langmuir model. }\end{array}$ & 42 \\
\hline
\end{tabular}




\begin{tabular}{|c|c|c|c|c|c|}
\hline No. & $\begin{array}{l}\text { Metal / } \\
\text { Medium }\end{array}$ & $\begin{array}{l}\text { Inhibitor / } \\
\text { Additives }\end{array}$ & Methods & Findings & Ref. \\
\hline & & & techniques & & \\
\hline 43 & $\begin{array}{l}\text { Graphene } \\
\text { oxide }(\mathrm{GO}) \\
\text { nanosheets }\end{array}$ & $\begin{array}{l}\text { Urtica Dioica } \\
\text { leaves }\end{array}$ & $\begin{array}{l}\text { Fourier transform } \\
\text { infrared } \\
\text { spectroscopy, high } \\
\text { resolution- } \\
\text { Transmission } \\
\text { electron microscopy, } \\
\text { field-emission } \\
\text { scanning electron } \\
\text { microscopy, UV- } \\
\text { visible spectroscopy, } \\
\text { and thermal } \\
\text { gravimetric analysis }\end{array}$ & $\begin{array}{l}\text { Physisorption mechanism, } \\
\text { intermolecular H-bonds. }\end{array}$ & 43 \\
\hline 44 & $\begin{array}{l}\text { Aluminum } \\
\text { in } \\
\text { hydrochloric } \\
\text { acid }\end{array}$ & $\begin{array}{c}\text { Murraya koenigii } \\
\text { leaves extract of } \\
\mathrm{pH}=3 \text { at } 303^{\circ} \mathrm{K} \text { to } \\
323^{\circ} \mathrm{K} \text {. }\end{array}$ & $\begin{array}{l}\text { Tafel polarization } \\
\text { and electrochemical } \\
\text { impedance } \\
\text { spectroscopy } \\
\text { techniques }\end{array}$ & $\begin{array}{l}\text { Anodic type of inhibitor, } \\
\text { Langmuir adsorption isotherm. }\end{array}$ & 44 \\
\hline 45 & $\begin{array}{c}\text { Stainless } \\
\text { steel 304 } \\
\text { (SS304) in } \\
2 \mathrm{~m} \\
\text { hydrochloric } \\
\text { acid }\end{array}$ & $\begin{array}{l}\text { Tectona grandis } \\
\text { leaf extract }\end{array}$ & $\begin{array}{l}\text { Potentiodynamic } \\
\text { polarization studies, } \\
\text { Fourier transform } \\
\text { infrared } \\
\text { spectroscopy }\end{array}$ & $\begin{array}{l}\text { Mixed type inhibitor, } \\
\text { physisorption and } \\
\text { chemisorptions processes. }\end{array}$ & 45 \\
\hline 46 & $\begin{array}{l}\text { Mild steel in } \\
3.5 \% \mathrm{NaCl}\end{array}$ & $\begin{array}{l}\text { Glycyrrhiza } \\
\text { glabra leaf } \\
\text { extract }\end{array}$ & $\begin{array}{c}\text { Polarization } \\
\text { measurements, UV, } \\
\text { IR and SEM study }\end{array}$ & Mixed type of inhibitor & 46 \\
\hline 47 & $\begin{array}{l}\text { Mild Steel in } \\
1 \mathrm{M} \mathrm{HCl}\end{array}$ & $\begin{array}{c}\text { Bauhinia } \\
\text { tomentosa leaves } \\
\text { extract, } 308- \\
333^{\circ} \mathrm{K}\end{array}$ & $\begin{array}{c}\text { weight-loss method } \\
\text { and electrochemical } \\
\text { techniques, FT-IR, } \\
\text { UV, SEM and AFM } \\
\text { techniques }\end{array}$ & $\begin{array}{c}\text { Mixed-type inhibitor, } \\
\text { Langmuir adsorption isotherm. }\end{array}$ & 47 \\
\hline 48 & $\begin{array}{l}\text { Aluminium } \\
\text { in } 1 \mathrm{M} \\
\mathrm{H}_{2} \mathrm{SO}_{4}\end{array}$ & $\begin{array}{l}\text { Dryopteris } \\
\text { cochleata leaf } \\
\text { extracts }\end{array}$ & $\begin{array}{c}\text { Weight loss, } \\
\text { electrochemical } \\
\text { measurements, } X \text {-ray } \\
\text { diffraction and } \\
\text { scanning electron } \\
\text { microscopy }\end{array}$ & Mixed type inhibitor & 48 \\
\hline 49 & $\begin{array}{l}\text { Stainless } \\
\text { steel } 304 \text { in } \\
\text { acidic }\end{array}$ & $\begin{array}{l}\text { Thymus vulgaris } \\
\text { leaf extracts }\end{array}$ & $\begin{array}{l}\text { Electrochemical } \\
\text { impedance } \\
\text { spectroscopy, }\end{array}$ & $\begin{array}{l}\text { Positive corrosion inhibiting } \\
\text { properties. }\end{array}$ & 49 \\
\hline
\end{tabular}




\begin{tabular}{|c|c|c|c|c|c|}
\hline No. & $\begin{array}{l}\text { Metal / } \\
\text { Medium }\end{array}$ & $\begin{array}{l}\text { Inhibitor / } \\
\text { Additives }\end{array}$ & Methods & Findings & Ref. \\
\hline & solution & & $\begin{array}{l}\text { electrochemical } \\
\text { noise analysis and } \\
\text { density functional } \\
\text { theory }\end{array}$ & & \\
\hline 50 & $\begin{array}{l}\text { Carbon steel } \\
\text { in acidic } \\
\text { media }\end{array}$ & $\begin{array}{l}\text { Tetraclinis } \\
\text { articulata }(\text { Vahl }) \\
\text { masters leaves }\end{array}$ & $\begin{array}{c}\text { Electrochemical } \\
\text { polarization method }\end{array}$ & Mixed type inhibitor & 50 \\
\hline 51 & $\begin{array}{c}\text { Monel } 400 \\
\text { in } \\
\text { hydrochloric } \\
\text { solution }\end{array}$ & $\begin{array}{c}\text { Green leaves of } \\
\text { Mespilus } \\
\text { japonica, Ricinus } \\
\text { communis and } \\
\text { Vitis vinifera }\end{array}$ & $\begin{array}{l}\text { Electrochemical } \\
\text { impedance } \\
\text { spectroscopy and } \\
\text { potentiodynamic } \\
\text { polarization }\end{array}$ & $\begin{array}{l}\text { Langmiur isotherm, good } \\
\text { corrosion inhibitors. }\end{array}$ & 51 \\
\hline 52 & - & $\begin{array}{l}\text { Aqueous extract } \\
\text { of leaves of } \\
\text { Pancratium } \\
\text { Foetidum Pom }\end{array}$ & $\begin{array}{l}\text { Potentiodynamic } \\
\text { polarization, } \\
\text { electrochemical } \\
\text { impedance } \\
\text { spectroscopy and } \\
\text { weight loss studies }\end{array}$ & $\begin{array}{l}\text { Inhibition efficiency increases } \\
\text { with the increase in inhibitor } \\
\text { concentration }\end{array}$ & 52 \\
\hline 53 & $\begin{array}{l}\text { Mild steel in } \\
1 \mathrm{~N} \mathrm{HCl}\end{array}$ & $\begin{array}{l}\text { Schrebera } \\
\text { swietenioids } \\
\quad \text { leaves }\end{array}$ & $\begin{array}{l}\text { Mass loss method, } \\
\text { polarization } \\
\text { measurements and } \\
\text { electrochemical } \\
\text { impedance } \\
\text { spectroscopy, SEM } \\
\text { analysis, FTIR } \\
\text { studies }\end{array}$ & Mixed type inhibitor. & 53 \\
\hline 54 & $\begin{array}{l}\text { Aluminium } \\
\text { in } \\
\text { hydrochloric } \\
\text { acid }\end{array}$ & $\begin{array}{l}\text { Delonix regia } \\
\text { (Gulmohar) } \\
\quad \text { extract }\end{array}$ & $\begin{array}{c}\text { Weight loss, } \\
\text { Potentiodynamic } \\
\text { Polarization, } \\
\text { Electrochemical } \\
\text { Impedance } \\
\text { Spectroscopic (EIS) } \\
\text { techniques }\end{array}$ & $\begin{array}{c}\text { Langmuir adsorption isotherm, } \\
\text { Thermodynamic parameters } \\
\text { were calculated }\end{array}$ & 54 \\
\hline 55 & $\begin{array}{l}\mathrm{J} 55 \text { steel in } \\
3.5 \% \mathrm{NaCl} \\
\text { solution } \\
\text { saturated } \\
\text { with } \mathrm{CO}_{2}\end{array}$ & $\begin{array}{l}\text { Ilex kudingcha } \\
\text { C.J. Tseng } \\
\text { (Kudingcha) } \\
\text { leaves extract }\end{array}$ & $\begin{array}{c}\text { Fourier transform } \\
\text { infrared } \\
\text { spectroscopy (FTIR), } \\
\text { potentiodynamic } \\
\text { polarization, } \\
\text { electrochemical } \\
\text { impedance } \\
\text { spectroscopy (EIS) } \\
\text { and scanning }\end{array}$ & $\begin{array}{c}\text { Langmuir, Frumkin and } \\
\text { Temkin isotherms, mixed type } \\
\text { inhibitor. }\end{array}$ & 55 \\
\hline
\end{tabular}




\begin{tabular}{|c|c|c|c|c|c|}
\hline No. & $\begin{array}{l}\text { Metal / } \\
\text { Medium }\end{array}$ & $\begin{array}{l}\text { Inhibitor / } \\
\text { Additives }\end{array}$ & Methods & Findings & Ref. \\
\hline & & & $\begin{array}{l}\text { electron microscopy } \\
\text { (SEM) methods }\end{array}$ & & \\
\hline 56 & $\begin{array}{l}\text { Mild steel in } \\
\text { aqueous } \\
\text { hydrochloric } \\
\text { acid solution }\end{array}$ & $\begin{array}{c}\text { Jatropha Curcas } \\
\text { leaves }\end{array}$ & $\begin{array}{l}\text { Gravimetric and } \\
\text { thermometric } \\
\text { techniques }\end{array}$ & Langmuir isotherm model & 56 \\
\hline 57 & $\begin{array}{l}\text { Low-alloy } \\
\text { steel in } \\
\text { acidic } \\
\text { medium }\end{array}$ & $\begin{array}{c}\text { Aloe Vera leaves } \\
\text { extract }\end{array}$ & $\begin{array}{c}\text { Gravimetric analysis, } \\
\text { potentiostatic } \\
\text { polarization } \\
\text { measurements, } \\
\text { electrochemical } \\
\text { impedance } \\
\text { spectroscopy and } \\
\text { quantum analysis }\end{array}$ & $\begin{array}{l}\text { Langmuir adsorption isotherm, } \\
\text { Dubinin-Radushkevich } \\
\text { isotherm, Mixed type inhibitor }\end{array}$ & 57 \\
\hline 58 & $\begin{array}{c}\text { API 5L } \\
\text { Carbon steel } \\
\text { in } 0.5 \mathrm{M} \\
\mathrm{H}_{2} \mathrm{SO}_{4}\end{array}$ & $\begin{array}{l}\text { Thyme leaves } \\
\text { hydroalcoholic } \\
\text { extract }\end{array}$ & $\begin{array}{c}\text { Tafel polarization } \\
\text { and electrochemical } \\
\text { impedance } \\
\text { spectroscopy }\end{array}$ & Mixed type inhibitor & 58 \\
\hline 59 & $\begin{array}{l}\text { Copper in } \\
\text { neutral } \\
\text { medium }\end{array}$ & $\begin{array}{l}\text { Azadirachta } \\
\text { indica leaves } \\
\quad \text { extract }\end{array}$ & $\begin{array}{l}\text { Electrochemical } \\
\text { impedance } \\
\text { spectroscopy, } \\
\text { potentiodynamic } \\
\text { polarization studies, } \\
\text { scanning electron } \\
\text { microscopy }\end{array}$ & $\begin{array}{l}\text { Good corrosion inhibiting } \\
\text { properties }\end{array}$ & 59 \\
\hline 60 & $\begin{array}{l}\text { Aluminium } \\
\text { in } 2 \mathrm{M} \\
\text { phosphoric } \\
\text { acid }\end{array}$ & $\begin{array}{c}\text { Mentha pulegium } \\
\text { leaves }\end{array}$ & $\begin{array}{l}\text { Potentiodynamic } \\
\text { polarization and } \\
\text { electrochemical } \\
\text { impedance } \\
\text { spectroscopy (EIS) }\end{array}$ & Cathodic inhibitor & 60 \\
\hline
\end{tabular}

Table 4. Outcomes of the study

\begin{tabular}{|c|c|c|c|c|c|}
\hline No. & $\begin{array}{c}\text { Metal / } \\
\text { medium }\end{array}$ & $\begin{array}{c}\text { Inhibitor / } \\
\text { Additives }\end{array}$ & Methods & Findings & Ref. \\
\hline 61 & $\begin{array}{c}\text { Steel in } \\
1 \mathrm{M} \mathrm{HCl}\end{array}$ & $\begin{array}{c}\text { Morus Alba } \\
\text { Pendula leaves } \\
\text { extract (MAPLE) }\end{array}$ & $\begin{array}{c}\text { Electrochemical } \\
\text { impedance } \\
\text { spectroscopy (EIS) } \\
\text { and polarization test, } \\
\text { (AFM), (FT-IR), } \\
\text { UV-visible analysis }\end{array}$ & $\begin{array}{c}\text { High inhibition efficiency } \\
\text { value of 93\%. A synergistic } \\
\text { effect was observed between } \\
\text { KI and MAPLE with optimum } \\
\text { concentration of } 0.4 \mathrm{~g} / \mathrm{L} \\
\text { MAPLE }+10 \mathrm{mM} \text { KI. } \\
\text { Adsorption of extract on the }\end{array}$ & 61 \\
\hline
\end{tabular}




\begin{tabular}{|c|c|c|c|c|c|}
\hline No. & $\begin{array}{l}\text { Metal / } \\
\text { medium }\end{array}$ & $\begin{array}{l}\text { Inhibitor / } \\
\text { Additives }\end{array}$ & Methods & Findings & Ref. \\
\hline & & & & steel surface. & \\
\hline 62 & $\begin{array}{l}\text { Carbon steel } \\
\text { in } 1 \mathrm{M} \mathrm{HCl}\end{array}$ & $\begin{array}{c}\text { Ziziphus jujuba } \\
\text { leaves extract }\end{array}$ & $\begin{array}{l}\text { Weight loss } \\
\text { measurements, } \\
\text { gasometric method, } \\
\text { potentiodynamic } \\
\text { polarization curves } \\
\text { and electrochemical } \\
\text { impedance } \\
\text { spectroscopy } \\
\text { methods }\end{array}$ & $\begin{array}{l}\text { Inhibition efficiency increased } \\
\text { with extracts concentration. } \\
\text { The maximum inhibition } \\
\text { efficiency of } 82.2 \% \text { was } \\
\text { obtained. ZJL extract acts as a } \\
\text { mixed-type. Langmuir } \\
\text { adsorption isotherm. }\end{array}$ & 62 \\
\hline 63 & $\begin{array}{c}\text { Carbon steel } \\
\text { in } 1 \mathrm{M} \mathrm{HCl} \\
\text { and } 0.5 \mathrm{M} \\
\mathrm{H}_{2} \mathrm{SO}_{4}\end{array}$ & $\begin{array}{l}\text { Coriandrum } \\
\text { sativum leaves } \\
\text { extract (CSL) }\end{array}$ & $\begin{array}{l}\text { Potentiodynamic } \\
\text { polarization, } \\
\text { electrochemical } \\
\text { impedance } \\
\text { spectroscopy (EIS), } \\
\text { weight loss } \\
\text { techniques and } \\
\text { scanning electron } \\
\text { microscopy analysis }\end{array}$ & $\begin{array}{l}\text { The inhibition efficiency } \\
\text { increased with the increase of } \\
\text { the concentration of the extract } \\
\text { and decreased with the increase } \\
\text { in temperature. Langmuir } \\
\text { adsorption isotherm model. }\end{array}$ & 63 \\
\hline 64 & $\begin{array}{l}\text { Mild steel in } \\
1 \mathrm{~N} \mathrm{HCl} \\
\text { medium }\end{array}$ & $\begin{array}{c}\text { Balsamodendron } \\
\text { Caudatum (BC) } \\
\text { leaves }\end{array}$ & $\begin{array}{l}\text { Weight loss, } \\
\text { potentiodynamic } \\
\text { polarization methods } \\
\text { and EIS, FTIR }\end{array}$ & $\begin{array}{l}\text { Inhibition efficiency (\% IE) was } \\
\text { proportional to inhibitor } \\
\text { concentration. Mixed type } \\
\text { inhibitors. The surface } \\
\text { morphology was determined by } \\
\text { Scanning Electron Microscopy. }\end{array}$ & 64 \\
\hline 65 & $\begin{array}{l}\text { Copper in } \\
\text { nitric acid } \\
\text { solution }\end{array}$ & Lawsonia extract & $\begin{array}{l}\text { Weight loss, } \\
\text { potentiodynamic } \\
\text { polarization and } \\
\text { electrochemical } \\
\text { impedance } \\
\text { spectroscopic } \\
\text { methods }\end{array}$ & $\begin{array}{l}\text { Inhibition efficiency is } \\
\text { enhanced with increase of } \\
\text { inhibitor concentration and } \\
\text { decrease with increase in } \\
\text { temperature. Langmuir } \\
\text { adsorption isotherm in all } \\
\text { tested systems. Mixed type } \\
\text { inhibitor. Inhibition efficiency } \\
\text { was obtained up to } 98 \% \text {. }\end{array}$ & 65 \\
\hline 66 & $\begin{array}{l}\text { Mild steel in } \\
1 \mathrm{M} \mathrm{H}_{2} \mathrm{SO}_{4}\end{array}$ & Rhus verniciflua & $\begin{array}{l}\text { Fourier transform } \\
\text { infrared } \\
\text { spectroscopy, } \\
\text { electrochemical } \\
\text { impedance } \\
\text { spectroscopy, and } \\
\text { potentiodynamic } \\
\text { polarization } \\
\text { measurements. SEM, }\end{array}$ & $\begin{array}{l}\text { The inhibition efficiency was } \\
\text { found to increase with an } \\
\text { increase in the inhibitor } \\
\text { concentration. On the other } \\
\text { hand, the inhibition efficiency } \\
\text { decreased with an increase in } \\
\text { the temperature. Langmuir, } \\
\text { Temkin, and } \\
\text { El-Awady isotherms. }\end{array}$ & 66 \\
\hline
\end{tabular}




\begin{tabular}{|c|c|c|c|c|c|}
\hline No. & $\begin{array}{l}\text { Metal / } \\
\text { medium }\end{array}$ & $\begin{array}{l}\text { Inhibitor / } \\
\text { Additives }\end{array}$ & Methods & Findings & Ref. \\
\hline & & & EDAX & & \\
\hline 67 & $\begin{array}{l}\text { Mild steel in } \\
\mathrm{HCl} \text { solution }\end{array}$ & $\begin{array}{c}\text { Aquilaria } \\
\text { malaccensis }\end{array}$ & $\begin{array}{l}\text { Gravimetric and } \\
\text { electrochemical } \\
\text { methods }\end{array}$ & $\begin{array}{l}\text { Inhibit corrosion by as much as } \\
94.49 \% \text { at the concentration of } \\
1500 \text { ppm. The leaf extract } \\
\text { acted as a mixed-type, but } \\
\text { predominantly cathodic } \\
\text { inhibitor for the } \\
\text { potentiodynamic polarization } \\
\text { measurement. Langmuir } \\
\text { adsorption isotherm model. }\end{array}$ & 67 \\
\hline 68 & $\begin{array}{l}\text { Steel-rebar } \\
\text { in } \mathrm{H}_{2} \mathrm{SO}_{4}\end{array}$ & $\begin{array}{c}\text { Anthocleista } \\
\text { djalonensis and } \\
\mathrm{Na}_{2} \mathrm{Cr}_{2} \mathrm{O}_{7}\end{array}$ & $\begin{array}{c}\text { Steel-reinforced } \\
\text { concrete samples } \\
\text { immersed in the test- } \\
\text { system, from which } \\
\text { macrocell corrosion } \\
\text { measurements were } \\
\text { obtained and } \\
\text { analysed as per } \\
\text { ASTM G109-99a }\end{array}$ & $\begin{array}{l}\text { Results showed that only the } \\
3.33 \mathrm{~g} / \mathrm{L} \text { Anthocleista } \\
\text { djalonensis, among the equal- } \\
\text { mass models of leaf extract and } \\
\text { the chemical admixtures, was } \\
\text { outperformed by the } 3.33 \mathrm{~g} / \mathrm{L} \\
\mathrm{Na}_{2} \mathrm{Cr}_{2} \mathrm{O}_{7} \text { in total corrosion } \\
\text { reduction effects. In the study, } \\
5.00 \mathrm{~g} / \mathrm{L} \text { Anthocleista } \\
\text { djalonensis exhibited optimal } \\
\text { effectiveness, } \mathrm{n}=93.77 \% \text {, on } \\
\text { the total-corrosion effect of } \\
\text { concrete steel-reinforcement. }\end{array}$ & 68 \\
\hline 69 & $\begin{array}{l}\text { Mild steel in } \\
\mathrm{H}_{2} \mathrm{SO}_{4} \\
\text { medium }\end{array}$ & $\begin{array}{l}\text { Tribulus } \\
\text { terrestris extracts }\end{array}$ & $\begin{array}{l}\text { Mass loss, EIS and } \\
\text { polarization, SEM }\end{array}$ & $\begin{array}{l}\text { Good corrosion inhibition } \\
\text { efficiency, Langmuir } \\
\text { adsorption isotherm }\end{array}$ & 69 \\
\hline 70 & $\begin{array}{l}\text { Mild steel in } \\
8 \% \mathrm{H}_{2} \mathrm{SO}_{4}\end{array}$ & $\begin{array}{c}\text { Withania } \\
\text { somnifera extract }\end{array}$ & $\begin{array}{c}\text { By using } \\
\text { polarization } \\
\text { measurements, UV, } \\
\text { IR and weight loss } \\
\text { study }\end{array}$ & $\begin{array}{l}\text { Plant extract acts as better } \\
\text { inhibitor on increasing its } \\
\text { concentration. }\end{array}$ & 70 \\
\hline 71 & $\begin{array}{c}\text { Mild steel in } \\
0.5 \mathrm{M} \\
\mathrm{H}_{2} \mathrm{SO}_{4} \\
\text { solution }\end{array}$ & $\begin{array}{c}\text { Thymus } \\
\text { Algeriensis }\end{array}$ & $\begin{array}{l}\text { Investigated by } \\
\text { electrochemical } \\
\text { studies }\end{array}$ & $\begin{array}{l}\text { Investigated by } \\
\text { electrochemical studies in the } \\
\text { presence of different } \\
\text { concentrations of TAE \& TAO } \\
\text { ranging from } 0.25 \mathrm{~g} / \mathrm{L} \text { to } 2 \mathrm{~g} / \mathrm{L} \text {. } \\
\text { TAE and TAO behave as } \\
\text { mixed type inhibitors. }\end{array}$ & 71 \\
\hline 72 & $\begin{array}{l}\text { Mild steel in } \\
2 \mathrm{M} \mathrm{H}_{2} \mathrm{SO}_{4} \\
\text { solution }\end{array}$ & $\begin{array}{c}\text { By Nicotiana } \\
\text { tabacum extract }\end{array}$ & $\begin{array}{l}\text { Studied by weight } \\
\text { loss method }\end{array}$ & $\begin{array}{l}\text { The inhibition efficiency (\% IE) } \\
\text { and surface coverage }(\theta) \text { of } N \text {. } \\
\text { tabacum extract increased with } \\
\text { increase in inhibitor }\end{array}$ & 72 \\
\hline
\end{tabular}




\begin{tabular}{|c|c|c|c|c|c|}
\hline No. & $\begin{array}{l}\text { Metal / } \\
\text { medium }\end{array}$ & $\begin{array}{l}\text { Inhibitor / } \\
\text { Additives }\end{array}$ & Methods & Findings & Ref. \\
\hline & & & & $\begin{array}{l}\text { concentration but decreased } \\
\text { with increasing the } \\
\text { temperature. Langmuir's } \\
\text { adsorption isotherm. The free } \\
\text { energy value }\left(\Delta \mathrm{G}_{\mathrm{ads}}\right) \text { indicated } \\
\text { that the adsorption of inhibitor } \\
\text { molecules was typical of } \\
\text { physisorption. Quantum } \\
\text { chemical parameters such as } \\
\text { highest occupied molecular } \\
\text { orbital energy }\left(E_{\mathrm{HOMO}}\right) \text {, lowest } \\
\text { unoccupied molecular orbital } \\
\text { energy }\left(E_{\mathrm{LuMO}}\right) \text {, energy gap } \\
\text { ( } \Delta \mathrm{E}) \text {, dipole moment }(\mu) \text { and } \\
\text { Mulliken charges were } \\
\text { calculated. Quantum chemical } \\
\text { calculations }\end{array}$ & \\
\hline 73 & $\begin{array}{l}\text { Carbon steel } \\
\text { in } 1 \mathrm{M} \\
\text { sulphuric } \\
\text { acid }\end{array}$ & $\begin{array}{c}\text { Eucalyptus } \\
\text { globulus Leaves } \\
\text { Cultivated in } \\
\text { Tunisia Arid } \\
\text { Zones }\end{array}$ & $\begin{array}{l}\text { Weight loss } \\
\text { measurements and } \\
\text { different } \\
\text { electrochemical } \\
\text { methods. }\end{array}$ & $\begin{array}{l}\text { This compound acts as a } \\
\text { mixed-type inhibitor. As the } \\
\text { inhibitor concentration } \\
\text { increased, the charge transfer } \\
\text { resistance of carbon steel } \\
\text { increased and double layer } \\
\text { capacitance decreased. The } \\
\text { results of weight loss } \\
\text { measurements were in good } \\
\text { agreement with other } \\
\text { electrochemical methods } \\
\text { results. adsorption obeys the } \\
\text { Langmuir isotherm. }\end{array}$ & 73 \\
\hline 74 & $\begin{array}{l}\text { Aluminium } \\
\text { in } \\
\text { hydrochloric } \\
\text { acid }\end{array}$ & $\begin{array}{c}\text { Delonix regia } \\
\text { (Gulmohor) } \\
\text { extract }\end{array}$ & $\begin{array}{c}\text { Weight loss, } \\
\text { polarization, EIS }\end{array}$ & $\begin{array}{l}\text { As inhibitor concentration } \\
\text { increases corrosion rate } \\
\text { decreases while percentage of } \\
\text { inhibition efficiency (IE) } \\
\text { increases. Obeys Langmuir } \\
\text { adsorption isotherm. inhibitor } \\
\text { acts as mixed type. }\end{array}$ & 74 \\
\hline 75 & $\begin{array}{l}\text { Steel in } \\
1 \mathrm{M} \mathrm{HCl}\end{array}$ & $\begin{array}{l}\text { Extracts of } \\
\text { Diospyros } \\
\text { kaki L.f. } \\
\text { (persimmon) } \\
\quad \text { leaves }\end{array}$ & $\begin{array}{l}\text { Investigated, by } \\
\text { using weight loss } \\
\text { and potentiodynamic } \\
\text { polarisation } \\
\text { techniques }\end{array}$ & $\begin{array}{l}\text { The highest efficiency was } \\
94.3 \% \text {. The extracts inhibit } \\
\text { corrosion mainly by an } \\
\text { adsorption mechanism. Mixed- } \\
\text { type inhibitors. In addition, the } \\
\text { extracts were screened for } \\
\text { antibacterial activity against oil }\end{array}$ & 75 \\
\hline
\end{tabular}




\begin{tabular}{|c|c|c|c|c|c|}
\hline No. & $\begin{array}{l}\text { Metal / } \\
\text { medium }\end{array}$ & $\begin{array}{l}\text { Inhibitor / } \\
\text { Additives }\end{array}$ & Methods & Findings & Ref. \\
\hline & & & & $\begin{array}{c}\text { field microorganisms, and they } \\
\text { showed good to moderate } \\
\text { activity against SRB, IB, and } \\
\text { TGB }\end{array}$ & \\
\hline 76 & $\begin{array}{l}\text { Steel } \\
\text { materials in } \\
\text { hydrochloric } \\
\text { acid }\end{array}$ & $\begin{array}{l}\text { Michelia alba } \\
\text { leaves extract }\end{array}$ & $\begin{array}{c}\text { Investigated by } \\
\text { potentiodynamic } \\
\text { polarization, } \\
\text { scanning electron } \\
\text { microscopy (SEM), } \\
\text { Fourier transform } \\
\text { infrared } \\
\text { spectroscopy (FTIR) } \\
\text { and quantum } \\
\text { chemical } \\
\text { calculations }\end{array}$ & $\begin{array}{l}\text { The results showed that MALE } \\
\text { acted as a highly-efficient } \\
\text { mixed-type inhibitor for all } \\
\text { steels and increasing } \\
\text { temperatures benefited its } \\
\text { corrosion inhibition. The } \\
\text { adsorption of MALE on steel } \\
\text { surfaces obeyed a Langmuir } \\
\text { adsorption isotherm. Quantum } \\
\text { chemical calculation results } \\
\text { provided reasonable theoretical } \\
\text { explanation for the inhibition } \\
\text { property of MALE. }\end{array}$ & 76 \\
\hline 77 & $\begin{array}{l}\text { Mild steel in } \\
\text { aqueous } \\
\text { medium }\end{array}$ & $\begin{array}{l}\text { Citrus sinensis } L \text {. } \\
\quad \text { leaf extract }\end{array}$ & $\begin{array}{l}\text { Using weight loss } \\
\text { method, pH, UV- } \\
\text { Vis, and FT-IR. To } \\
\text { prepare the extract, } \\
\text { the shade-dried leaf } \\
\text { of Citrus sinensis L. } \\
\text { FT-IR and UV }\end{array}$ & $\begin{array}{l}\text { Citrus sinensis } L \text {. leaf extract } \\
\text { have several bioactive } \\
\text { compounds, can be used as } \\
\text { corrosion inhibitor and such } \\
\text { rare morphovariant should be } \\
\text { conserved in nature. }\end{array}$ & 77 \\
\hline 78 & $\begin{array}{l}\text { Mild steel in } \\
\text { phosphoric } \\
\text { acid }\end{array}$ & $\begin{array}{l}\text { Psidium guajava } \\
\text { leaf extract }\end{array}$ & $\begin{array}{l}\text { Investigated by } \\
\text { weight loss, } \\
\text { potentiodynamic } \\
\text { polarization and } \\
\text { electrochemical } \\
\text { impedance } \\
\text { spectroscopy } \\
\text { techniques }\end{array}$ & $\begin{array}{l}\text { Inhibition efficiency increases } \\
\text { with inhibitor concentration up } \\
\text { to } 800 \mathrm{ppm} \text { and decreases } \\
\text { slightly at } 1200 \mathrm{ppm} \text {. The } \\
\text { adsorption obeys both the } \\
\text { Langmuir and the Temkin } \\
\text { adsorption isotherm equations. } \\
\text { the inhibitor acted as a mixed- } \\
\text { type inhibitor. }\end{array}$ & 78 \\
\hline 79 & $\begin{array}{l}\text { Steel in } \\
\text { brine } \\
\text { solution } \\
\text { saturated } \\
\text { with } \mathrm{CO}_{2}\end{array}$ & Olive leaf extract & & $\begin{array}{l}\text { Olive leaf extract acts as good } \\
\text { green inhibitor. }\end{array}$ & 79 \\
\hline
\end{tabular}




\begin{tabular}{|c|c|c|c|c|c|}
\hline No. & $\begin{array}{l}\text { Metal / } \\
\text { medium }\end{array}$ & $\begin{array}{c}\text { Inhibitor / } \\
\text { Additives }\end{array}$ & Methods & Findings & Ref. \\
\hline 80 & $\begin{array}{l}\text { Carbon steel } \\
\text { in } 1 \mathrm{M} \\
\text { hydrochloric } \\
\text { acid }\end{array}$ & $\begin{array}{c}\text { Date palm } \\
\text { (Phoenix } \\
\text { dactylifera) leaf } \\
\text { extract }\end{array}$ & $\begin{array}{l}\text { Investigated using } \\
\text { weight loss } \\
\text { measurements, linear } \\
\text { and potentiodynamic } \\
\text { polarization curves, } \\
\text { electrochemical } \\
\text { impedance } \\
\text { spectroscopy and } \\
\text { scanning electron } \\
\text { microscopy }\end{array}$ & $\begin{array}{l}\text { The inhibition efficiency } \\
\text { increased with increase in the } \\
\text { concentration of the inhibitor } \\
\text { but decreased with increase in } \\
\text { temperature. Inhibition } \\
\text { efficiency also was found to } \\
\text { increase as immersion time } \\
\text { increased. Langmuir isotherm. } \\
\text { Practical implications - Date } \\
\text { palm leaf extract (DPLE) is an } \\
\text { effective inhibitor at room } \\
\text { temperature and can be used to } \\
\text { protect plain carbon steel from } \\
\text { corrosion in HCl solution. } \\
\text { Originality/value - This study } \\
\text { provides new information on } \\
\text { the inhibiting characteristics of } \\
\text { DPLE under specified } \\
\text { conditions. The } \\
\text { environmentally friendly } \\
\text { inhibitor could find possible } \\
\text { applications in metal surface } \\
\text { anodizing and acid pickling } \\
\text { processes. }\end{array}$ & 80 \\
\hline
\end{tabular}

Table 5. Outcomes of the study

\begin{tabular}{|c|c|c|c|c|c|}
\hline No. & $\begin{array}{l}\text { Metal / } \\
\text { medium }\end{array}$ & $\begin{array}{l}\text { Inhibitor / } \\
\text { Additives }\end{array}$ & Methods & Findings & Ref. \\
\hline 81 & $\begin{array}{l}\text { Stainless } \\
\text { steel in } 1 \mathrm{M} \\
\mathrm{H}_{2} \mathrm{SO}_{4}\end{array}$ & $\begin{array}{c}\text { Aloe Vera leaf } \\
\text { extract }\end{array}$ & $\begin{array}{c}\text { Electrochemical } \\
\text { techniques, } \\
\text { electrochemical } \\
\text { noise (EN), scanning } \\
\text { electron microscope }\end{array}$ & $\begin{array}{l}\text { The results of linear } \\
\text { polarization and } \\
\text { electrochemical impedance } \\
\text { spectroscopy proved the } \\
\text { effectiveness of Aloe Vera } \\
\text { extract as concentration } \\
\text { increased. Employing EN, } \\
\text { different aspects like transient } \\
\text { analysis, noise resistant and } \\
\text { characteristic charge were } \\
\text { characterized. }\end{array}$ & 81 \\
\hline 82 & $\begin{array}{l}\text { Mild steel in } \\
1 \mathrm{M} \\
\text { hydrochloric }\end{array}$ & $\begin{array}{c}\text { Pistacia lentiscus } \\
\text { L. leaves } \\
\text { essential oil }\end{array}$ & $\begin{array}{l}\text { Weight loss, } \\
\text { Electrochemical } \\
\text { Impedance }\end{array}$ & $\begin{array}{l}\text { Inhibition was found to } \\
\text { increase with increasing } \\
\text { concentration of } P \text {. lentiscus }\end{array}$ & 82 \\
\hline
\end{tabular}




\begin{tabular}{|c|c|c|c|c|c|}
\hline No. & $\begin{array}{l}\text { Metal / } \\
\text { medium }\end{array}$ & $\begin{array}{l}\text { Inhibitor / } \\
\text { Additives }\end{array}$ & Methods & Findings & Ref. \\
\hline & acid solution & & $\begin{array}{c}\text { Spectroscopy (EIS) } \\
\text { and Tafel } \\
\text { polarization curves }\end{array}$ & $\begin{array}{l}\text { essential oil to attain } 90.8 \% \text { at } \\
2.56 \mathrm{~g} / \mathrm{L} \text { of }(\mathrm{PLL} \text { ) at } 303 \mathrm{~K} \text {. } \\
\text { The effect of temperature on } \\
\text { the system followed the } \\
\text { kinetic/thermodynamic model } \\
\text { of El-Awady et al. in the } \\
\text { temperature range from } 303 \text { to } \\
333 \mathrm{~K} \text {. The calculated } \\
\Delta \mathrm{G}^{\circ} \text { adsvalue showed that the } \\
\text { corrosion inhibition of the } \\
\text { carbon steel in } 1 \mathrm{M} \mathrm{HCl} \text { is } \\
\text { mainly controlled by a } \\
\text { physisorption process. }\end{array}$ & \\
\hline 83 & $\begin{array}{c}\text { Mild steel in } \\
2.0 \mathrm{M} \mathrm{HCl} \\
\text { and } 1.0 \mathrm{M} \\
\mathrm{H}_{2} \mathrm{SO}_{4} \\
\text { solutions }\end{array}$ & $\begin{array}{l}\text { Gundelia } \\
\text { tournefortii }\end{array}$ & $\begin{array}{l}\text { Weight loss } \\
\text { measurement, } \\
\text { potentiodynamic } \\
\text { polarization and } \\
\text { electrochemical } \\
\text { impedance } \\
\text { spectroscopy (EIS) }\end{array}$ & $\begin{array}{l}\text { The inhibition efficiency was } \\
\text { found to increase with increase } \\
\text { of the inhibitor concentrations } \\
\text { due to the adsorption of the } \\
\text { inhibitor molecules on the } \\
\text { metal surface. In addition, it } \\
\text { was established the Langmuir } \\
\text { adsorption isotherm fits well } \\
\text { with the experimental data. } \\
\text { The inhibition efficiency was } \\
\text { found to be } 93 \% \text { and } 90 \% \text { at } \\
150 \text { ppm in } 2.0 \mathrm{M} \text { HCl and } \\
1.0 \mathrm{M} \mathrm{H}_{2} \mathrm{SO}_{4} \text { respectively. } \\
\text { Cathodic and anodic } \\
\text { polarization curves show that } \\
\text { G. } \text { tournefortii extract is a } \\
\text { mixed-type inhibitor in both } \\
\text { acidic media. EIS showed that } \\
\text { the charge transfer controls the } \\
\text { corrosion process. The effect } \\
\text { of temperature on the } \\
\text { inhibition efficiency was } \\
\text { studied. }\end{array}$ & 83 \\
\hline 84 & $\begin{array}{l}\text { Cast iron } \\
\text { surface in } \\
1 \mathrm{M} \mathrm{HCl} \\
\text { medium }\end{array}$ & $\begin{array}{c}\text { Eleusine } \\
\text { aegyptiaca and } \\
\text { Croton rottleri } \\
\text { leaf extracts }\end{array}$ & $\begin{array}{l}\text { Weight loss and } \\
\text { Tafel polarization } \\
\text { and electrochemical } \\
\text { impedance } \\
\text { spectroscopy, FT-IR, } \\
\text { UV-vis, Wide-angle } \\
\text { X-ray diffraction and } \\
\text { SEM }\end{array}$ & $\begin{array}{l}\text { The inhibition efficiency } \\
\text { increased with inhibitor } \\
\text { concentrations. It was found } \\
\text { that the extracts acted as } \\
\text { mixed-type inhibitors. The } \\
\text { addition of halide additives } \\
\text { (KCl, KBr, and KI) on the } \\
\text { inhibition efficiency has also }\end{array}$ & 84 \\
\hline
\end{tabular}




\begin{tabular}{|c|c|c|c|c|c|}
\hline No. & $\begin{array}{l}\text { Metal / } \\
\text { medium }\end{array}$ & $\begin{array}{l}\text { Inhibitor / } \\
\text { Additives }\end{array}$ & Methods & Findings & Ref. \\
\hline & & & & $\begin{array}{l}\text { been investigated. The } \\
\text { adsorption of the inhibitors on } \\
\text { cast iron surface both in the } \\
\text { presence and absence of } \\
\text { halides follows the Langmuir } \\
\text { adsorption isotherm model. } \\
\text { The inhibiting nature of the } \\
\text { inhibitors was supported by } \\
\text { FT-IR, UV-vis, Wide-angle X- } \\
\text { ray diffraction and SEM } \\
\text { methods. }\end{array}$ & \\
\hline 85 & $\begin{array}{l}\text { Water } \\
\text { permeability } \\
\text { resistance } \\
\text { and } \\
\text { microstructure } \\
\text { of } \\
\text { reinforced } \\
\text { concrete } \\
\text { treated with } \\
\text { Bambusa } \\
\text { arundinacea } \\
\text { as green } \\
\text { corrosion } \\
\text { inhibitor }\end{array}$ & $\begin{array}{c}\text { Bambusa } \\
\text { Arundinacea } \\
\text { leaves compared } \\
\text { with that of } \\
\text { calcium nitrite } \\
\text { and ethanolamine }\end{array}$ & $\begin{array}{l}\text { Compressive } \\
\text { strength test, } \\
\text { durability } \\
\text { (permeability using } \\
\text { initial surface } \\
\text { absorption test } \\
\text { (ISAT) and field } \\
\text { emission scanning } \\
\text { electron microscopy } \\
\text { (FESEM)) for } 360 \\
\text { days exposure. }\end{array}$ & $\begin{array}{l}\text { Water absorption values of } \\
\text { steel reinforced concrete in the } \\
\text { presence of } \\
\text { Bambusa arundinacea inhibitor } \\
\text { were generally less than } 0.25, \\
0.17,0.1 \text { and } 0.07 \mathrm{~mL} / \mathrm{m}^{2} \mathrm{~s} \text { after } \\
10 \mathrm{~min}, 30 \mathrm{~min}, 1 \mathrm{~h} \text { and } 2 \mathrm{~h}, \\
\text { i.e., as required by ISAT } \\
\text { standard for low permeability } \\
\text { concrete. This might possibly } \\
\text { be due to the presence of } \\
\text { residual alkalinity of potassium } \\
\text { hydroxide (KOH) in the } \\
\text { concrete. KOH is adequate for } \\
\text { passivation and reduction of } \\
\text { permeability, which serves as a } \\
\text { chemical water barrier or } \\
\text { hydrophobic agent that } \\
\text { stabilizes calcium silicate } \\
\text { hydrates. }\end{array}$ & 85 \\
\hline 86 & $\begin{array}{l}\text { Mild steel in } \\
1 \mathrm{M} \mathrm{HCl}\end{array}$ & $\begin{array}{c}\text { Artemisia } \\
\text { Mesatlantica } \\
\text { essential oil } \\
\text { (AMEO) }\end{array}$ & $\begin{array}{l}\text { Weight loss } \\
\text { measurements }\end{array}$ & $\begin{array}{l}\text { The obtained results showed } \\
\text { that inhibition efficiency } \\
\text { increases with increasing } \\
\text { inhibitor concentration to attain } \\
91 \% \text { at } 2.76 \mathrm{~g} / \mathrm{L} \text { of AMEO at } \\
303 \mathrm{~K} \text {. Physical adsorption is } \\
\text { proposed for the corrosion } \\
\text { inhibition mechanism and the } \\
\text { process followed the } \\
\text { kinetic/thermodynamic model } \\
\text { of El-Awady et. al. in the } \\
\text { temperature range from } 303 \text { to } \\
343 \mathrm{~K} \text {. The adsorption and }\end{array}$ & 86 \\
\hline
\end{tabular}




\begin{tabular}{|c|c|c|c|c|c|}
\hline No. & $\begin{array}{l}\text { Metal / } \\
\text { medium }\end{array}$ & $\begin{array}{l}\text { Inhibitor / } \\
\text { Additives }\end{array}$ & Methods & Findings & Ref. \\
\hline & & & & $\begin{array}{l}\text { kinetic parameters for mild } \\
\text { steel/AMEO/1 M HCl system } \\
\text { were calculated from } \\
\text { experimental gravimetric data } \\
\text { and the interpretation of the } \\
\text { results are given. }\end{array}$ & \\
\hline 87 & $\begin{array}{c}\text { Aluminium } \\
\text { alloy in tea } \\
\text { extract in } \\
\mathrm{H}_{2} \mathrm{SO}_{4}\end{array}$ & Camellia sinensis & $\begin{array}{c}\text { Weight } \\
\text { loss/corrosion rate } \\
\text { and potential } \\
\text { measurement } \\
\text { techniques }\end{array}$ & $\begin{array}{l}\text { The extracts gave appreciable } \\
\text { corrosion inhibition } \\
\text { performance of aluminium at } \\
20 \text { and } 40 \% \text { concentrations } \\
\text { with the weight loss of } 236 \mathrm{mg} \\
(0.236 \mathrm{~g}) \text { and } 265 \mathrm{mg}(0.265 \mathrm{~g}) \\
\text { respectively. ANOVA test } \\
\text { confirmed the results at } 95 \% \\
\text { confidence, and further showed } \\
\text { that concentration of green tea } \\
\text { extract had greater effect on } \\
\text { potential and weight loss } \\
\text { measurements. The value of } \\
\text { Gibb's free energy of } \\
\text { adsorption obtained signified } \\
\text { that the mechanism of } \\
\text { adsorption of plant extract } \\
\text { molecules on the metal surface } \\
\text { was by physiosorption. }\end{array}$ & 87 \\
\hline 88 & $\begin{array}{l}\text { Aluminium } \\
\text { in } 1 \mathrm{~N} \\
\mathrm{NaOH} \\
\text { solution }\end{array}$ & $\begin{array}{l}\text { Vitex negundo } \\
\text { (VNL) leaves } \\
\text { extract }\end{array}$ & $\begin{array}{c}\text { Chemical and } \\
\text { electrochemical } \\
\text { measurements, SEM }\end{array}$ & $\begin{array}{l}\text { It was found that the inhibition } \\
\text { efficiency increased with } \\
\text { increase of VNL extract and } \\
\text { reached the maximum of } \\
72.6 \% \text { at } 900 \text { ppm of VNL } \\
\text { extract. The results obtained } \\
\text { from chemical and } \\
\text { electrochemical measurements } \\
\text { were in good agreement. The } \\
\text { potentiodynamic polarization } \\
\text { studies reveal that the VNL } \\
\text { extract act as mixed type } \\
\text { inhibitor. Adsorption of VNL } \\
\text { extract on the surface of } \\
\text { aluminium follows Langmuir } \\
\text { adsorption isotherm. The } \\
\text { surface characteristics of } \\
\text { inhibited and uninhibited } \\
\text { aluminium were investigated }\end{array}$ & 88 \\
\hline
\end{tabular}




\begin{tabular}{|c|c|c|c|c|c|}
\hline No. & $\begin{array}{l}\text { Metal / } \\
\text { medium }\end{array}$ & $\begin{array}{l}\text { Inhibitor / } \\
\text { Additives }\end{array}$ & Methods & Findings & Ref. \\
\hline & & & & $\begin{array}{l}\text { by scanning electron } \\
\text { microscope studies. }\end{array}$ & \\
\hline 89 & $\begin{array}{l}\text { Mild steel in } \\
1 \mathrm{M} \mathrm{HCl} \\
\text { medium }\end{array}$ & $\begin{array}{l}\text { Aquilaria } \\
\text { crassna leaves } \\
\text { extracts }\end{array}$ & $\begin{array}{l}\text { Gravimetric method } \\
\text { (weight loss } \\
\text { method), } \\
\text { electrochemical } \\
\text { methods } \\
\text { (electrochemical } \\
\text { impedance } \\
\text { spectroscopy (EIS) } \\
\text { and potentiodynamic } \\
\text { polarisation) and } \\
\text { scanning electron } \\
\text { microscope (SEM) }\end{array}$ & $\begin{array}{l}\text { The extracts were shown to } \\
\text { have good inhibition } \\
\text { efficiencies for the gravimetric } \\
\text { and electrochemical methods. } \\
\text { EIS analysis revealed that } \\
\text { increase in concentration } \\
\text { increases the charge transfer } \\
\text { resistance, thus increased } \\
\text { inhibition efficiency. The } \\
\text { potentiodynamic polarisation } \\
\text { measurements showed the } \\
\text { extracts acted as mixed-type } \\
\text { inhibitors with predominantly } \\
\text { cathodic effectiveness. SEM } \\
\text { techniques supported the } \\
\text { success of corrosion inhibition } \\
\text { with the presence of inhibitors } \\
\text { and the methanol extract best } \\
\text { fitted the Temkin adsorption } \\
\text { isotherm while the aqueous } \\
\text { extract best fitted the Langmuir } \\
\text { and Temkin adsorption } \\
\text { isotherms. The adsorption } \\
\text { mechanisms for both extracts } \\
\text { were mainly physisorption. }\end{array}$ & 89 \\
\hline 90 & $\begin{array}{l}\text { Mild steel in } \\
\text { hydrochloric } \\
\text { acid: the } \\
\text { case of oil } \\
\text { extract of } \\
\text { leaves of } \\
\text { Pistacia } \\
\text { lentiscus } \\
\text { from Saidia } \\
\text { Morocco }\end{array}$ & $\begin{array}{l}\text { Plants as a source } \\
\text { of green } \\
\text { corrosion } \\
\text { inhibitors: extract } \\
\text { of leaves of } \\
\text { Pistacia lentiscus } \\
\text { from Saidia } \\
\text { Morocco }\end{array}$ & $\begin{array}{c}\text { Gravimetric, } \\
\text { electrochemical } \\
\text { polarization and EIS } \\
\text { methods }\end{array}$ & $\begin{array}{c}\text { The inhibition efficiency } \\
\text { increases with increased } \\
\text { organic oil and extract } \\
\text { concentration to attain a } \\
\text { maximum value of } 96.34 \% \\
\text { and } 86.59 \% \text { at } 1 \mathrm{~g} / \mathrm{L} \text { for oil and } \\
\text { extract respectively. The study } \\
\text { reveals that oil, was dominated } \\
\text { by monoterpene hydrocarbons } \\
\text { (44.99\%) followed by } \\
\text { oxygenated monoterpenes } \\
\text { (13.66\%) and Sesquiterpene } \\
\text { hydrocarbons }(16.59 \%) \text {. } \\
\text { Among them, limonene } \\
\text { (18.92\%), } \alpha \text {-pinene }(13.94 \%), \\
\beta \text {-caryo phyllene }(6.93 \%) \text { and } \\
\text { terpinen-4-ol }(5.57 \%) \text { were }\end{array}$ & 90 \\
\hline
\end{tabular}




\begin{tabular}{|c|c|c|c|c|c|}
\hline No. & $\begin{array}{l}\text { Metal / } \\
\text { medium }\end{array}$ & $\begin{array}{l}\text { Inhibitor / } \\
\text { Additives }\end{array}$ & Methods & Findings & Ref. \\
\hline & & & & $\begin{array}{l}\text { identified as major } \\
\text { components. The Pistacia } \\
\text { lentiscus oil and extract acts a } \\
\text { mixed inhibitor and showed a } \\
\text { result of the growth in the } \\
\text { compactness of the protecting } \\
\text { film dressing. The organic oil } \\
\text { and extract adsorbs on the mild } \\
\text { steel surface according to a } \\
\text { Langmuir isotherm adsorption } \\
\text { model. }\end{array}$ & \\
\hline 91 & $\begin{array}{c}\text { Mild steel in } \\
\mathrm{HCl} \\
\text { medium }\end{array}$ & $\begin{array}{l}\text { Methanol leaf } \\
\text { extract of } \\
\text { Manihot } \\
\text { esculenta as } \\
\text { green corrosion } \\
\text { inhibitor }\end{array}$ & $\begin{array}{c}\text { The weight loss } \\
\text { method }\end{array}$ & $\begin{array}{l}\text { The best fit adsorption } \\
\text { isotherm model for methanol } \\
\text { leaf extract of Manihot } \\
\text { esculenta as green corrosion } \\
\text { inhibitor of corrosion of mild } \\
\text { steel in } 2 \mathrm{M} \text { HCl medium was } \\
\text { determined with the help of the } \\
\text { Adejo-Ekwenchi adsorption } \\
\text { isotherm. The inhibition } \\
\text { efficiency, \%IE, of the extract } \\
\text { was found to increase with } \\
\text { increase in both concentration } \\
\text { and temperature, suggestive } \\
\text { chemisorption. Going by the } \\
\text { values of \%IE, } R^{2} \text { and } \Delta G_{\text {ads }} \\
\text { this adsorption process would } \\
\text { have been thought to be } \\
\text { chemisorption and well fitted } \\
\text { into Langmuir, Freundlich and } \\
\text { Temkin isotherm models. } \\
\text { However, from the information } \\
\text { obtained through the Adejo- } \\
\text { Ekwenchi isotherm model the } \\
\text { adsorption process was } \\
\text { unambiguously resolved to be } \\
\text { physisorption and best fitted } \\
\text { into the Langmuir isotherm } \\
\text { model. }\end{array}$ & 91 \\
\hline 92 & $\begin{array}{l}\text { Mild steel in } \\
\text { sulfuric acid } \\
\text { solution }\end{array}$ & $\begin{array}{c}\text { Kleinia } \\
\text { grandiflora leaf } \\
\text { extract as a green } \\
\text { corrosion } \\
\text { inhibitor }\end{array}$ & $\begin{array}{c}\text { Mass-loss analysis, } \\
\text { potentiodynamic } \\
\text { polarization } \\
\text { measurements, } \\
\text { electrochemical }\end{array}$ & $\begin{array}{l}\text { The effect of temperature on } \\
\text { the corrosion behavior of mild } \\
\text { steel was studied in the range } \\
\text { of } 308 \text { to } 328 \mathrm{~K} \text {. The inhibition } \\
\text { efficiency was observed to }\end{array}$ & 92 \\
\hline
\end{tabular}




\begin{tabular}{|c|c|c|c|c|c|}
\hline No. & $\begin{array}{l}\text { Metal / } \\
\text { medium }\end{array}$ & $\begin{array}{l}\text { Inhibitor / } \\
\text { Additives }\end{array}$ & Methods & Findings & Ref. \\
\hline & & & $\begin{array}{c}\text { impedance } \\
\text { spectroscopy, } \\
\text { Fourier-transform } \\
\text { infrared } \\
\text { spectroscopy, } \\
\text { scanning electron } \\
\text { microscopy, UV- } \\
\text { visible spectroscopy, } \\
\text { and X-ray diffraction } \\
\text { analysis }\end{array}$ & $\begin{array}{l}\text { increase with increasing } \\
\text { concentration of the extract. } \\
\text { Polarization curves revealed } \\
\text { that the Kleinia grandiflora } \\
\text { leaf extract is a mixed } \\
\text { inhibitor. Impedance diagrams } \\
\text { revealed that an increase of } \\
\text { Kleinia grandiflora leaf extract } \\
\text { concentration increased the } \\
\text { charge transfer resistance and } \\
\text { decreased the double-layer } \\
\text { capacitance. The adsorption } \\
\text { process obeys Langmuir's } \\
\text { model, with a standard free } \\
\text { energy of adsorption ( } \Delta G_{\text {ads }} \text { of } \\
\text {-18.62 kJ/mol. The obtained } \\
\text { results indicate that the Kleinia } \\
\text { grandiflora leaf extract can } \\
\text { serve as an effective inhibitor } \\
\text { for the corrosion of mild steel } \\
\text { in a sulfuric acid medium. }\end{array}$ & \\
\hline 93 & $\begin{array}{l}\text { Mild steel in } \\
\text { acidic bath } \\
(1.2 \mathrm{~N} \mathrm{HCl} \\
\text { and } 1.2 \mathrm{~N} \\
\left.\mathrm{H}_{2} \mathrm{SO}_{4}\right)\end{array}$ & $\begin{array}{l}\text { Leaves of } \\
\text { Hibiscus } \\
\text { sabdariffa }\end{array}$ & & $\begin{array}{l}\text { The results of the present study } \\
\text { show that this compound has } \\
\text { decent inhibiting property for } \\
\text { mild steel corrosion in } 1.2 \mathrm{~N} \\
\mathrm{H}_{2} \mathrm{SO}_{4} \text { than } 1.2 \mathrm{~N} \mathrm{HCl} \text {. Four } \\
\text { sorption isotherms are tested } \\
\text { for the data, namely Langmuir, } \\
\text { Frumkin, Florry-Huggins, and } \\
\text { Langmuir-Freundlich } \\
\text { isotherms; of these the } \\
\text { Langmuir isotherm fits the data } \\
\text { well having correlation } \\
\text { coefficient over 0.99 in both } \\
\text { the acid environments. }\end{array}$ & 93 \\
\hline 94 & $\begin{array}{l}\text { Steel in } 1 \mathrm{M} \\
\mathrm{HCl} \text { solution }\end{array}$ & $\begin{array}{c}\text { Extract of } \\
\text { Mentha } \\
\text { rotundifolia } \\
\text { leaves (EMRL) }\end{array}$ & $\begin{array}{l}\text { Electrochemical } \\
\text { impedance } \\
\text { spectroscopy, Tafel } \\
\text { polarization } \\
\text { methods, and weight } \\
\text { loss measurements }\end{array}$ & $\begin{array}{l}\text { The results obtained revealed } \\
\text { that the inhibitor tested } \\
\text { differently reduced the kinetics } \\
\text { of the corrosion process of } \\
\text { steel. Its efficiency increases } \\
\text { with the concentration and } \\
\text { attained } 92.87 \% \text { at } 35 \% \text {. The } \\
\text { effect of temperature on the } \\
\text { corrosion behavior of steel in }\end{array}$ & 94 \\
\hline
\end{tabular}




\begin{tabular}{|c|c|c|c|c|c|}
\hline No. & $\begin{array}{l}\text { Metal / } \\
\text { medium }\end{array}$ & $\begin{array}{l}\text { Inhibitor / } \\
\text { Additives }\end{array}$ & Methods & Findings & Ref. \\
\hline & & & & $\begin{array}{l}1 \mathrm{M} \mathrm{HCl} \text { was also studied in } \\
\text { the range } 298 \text { and } 338 \mathrm{~K} \text {. The } \\
\text { thermodynamic data of } \\
\text { activation were determined. } \\
\text { Mentha rotundifolia extract is } \\
\text { adsorbed on the steel surface } \\
\text { according to a Langmuir } \\
\text { adsorption model. }\end{array}$ & \\
\hline 95 & $\begin{array}{l}\text { Mild steel in } \\
\mathrm{HCl} \text { solution }\end{array}$ & $\begin{array}{l}\text { UAE Rhazya } \\
\text { stricta Decne } \\
\text { extract }\end{array}$ & $\begin{array}{l}\text { Weight loss } \\
\text { measurements }\end{array}$ & $\begin{array}{l}\text { The aqueous Rhazya stricta } \\
\text { Decne leaves extract was found } \\
\text { to be a highly efficient } \\
\text { inhibitor for mild steel in } \\
1.0 \mathrm{M} \mathrm{HCl} \text { solution, reaching } \\
\text { about } 90 \text { per cent at } 2.0 \mathrm{~g} / \mathrm{L} \\
\text { and } 303 \mathrm{~K} \text {, a concentration } \\
\text { considered to be very } \\
\text { moderate. Even with one-tenth } \\
\text { of that concentration, } 0.2 \mathrm{~g} / \mathrm{L} \text {, } \\
\text { an inhibition of about } 82 \mathrm{per} \\
\text { cent was obtained at } 303 \mathrm{~K} \text {. } \\
\text { The rate of corrosion of the } \\
\text { mild steel in } 1.0 \mathrm{M} \text { HCl is a } \\
\text { function of the concentration of } \\
\text { the Rhazya stricta } \text { Decne } \\
\text { extract. This rate increases as } \\
\text { the concentration of the } R h a z y a \\
\text { stricta Decne extract is } \\
\text { increased. The percentage of } \\
\text { inhibition in the presence of } \\
\text { this inhibitor was decreased } \\
\text { with temperature which } \\
\text { indicates that physical } \\
\text { adsorption was the } \\
\text { predominant inhibition } \\
\text { mechanism because the } \\
\text { quantity of adsorbed inhibitor } \\
\text { decreases with increasing } \\
\text { temperature. }\end{array}$ & 95 \\
\hline 96 & $\begin{array}{l}\text { Carbon steel } \\
\text { in acidic } \\
\text { media }\end{array}$ & $\begin{array}{c}\text { Opuntia ficus } \\
\text { extract }\end{array}$ & $\begin{array}{l}\text { Weight loss } \\
\text { determinations, } \\
\text { surface studies, } \\
\text { electrochemical } \\
\text { impedance } \\
\text { spectroscopy and }\end{array}$ & $\begin{array}{l}\text { It was observed that Opuntia } \\
\text { ficus-indica extract can } \\
\text { decrease the corrosion rate of } \\
\mathrm{CS} \text {, and its efficiency increases } \\
\text { with increasing concentration } \\
\text { up to } 1,000 \text { ppm and with time, }\end{array}$ & 96 \\
\hline
\end{tabular}




\begin{tabular}{|c|c|c|c|c|c|}
\hline No. & $\begin{array}{l}\text { Metal / } \\
\text { medium }\end{array}$ & $\begin{array}{l}\text { Inhibitor / } \\
\text { Additives }\end{array}$ & Methods & Findings & Ref. \\
\hline & & & $\begin{array}{l}\text { potentiodynamic } \\
\text { polarization }\end{array}$ & $\begin{array}{l}\text { but decreases with increasing } \\
\text { the temperature from } 25 \text { to } \\
600^{\circ} \mathrm{C} \text {. The inhibitory activity } \\
\text { is due to the presence of } \\
\text { phenolic compounds in its } \\
\text { chemical structure. Research } \\
\text { limitations/implications: The } \\
\text { work was done under static } \\
\text { conditions, whereas in acid } \\
\text { cleaning conditions, there is a } \\
\text { dynamic system. However, the } \\
\text { findings may apply to both the } \\
\text { systems. }\end{array}$ & \\
\hline 97 & $\begin{array}{c}\text { Mild steel in } \\
0.5 \mathrm{M} \mathrm{HCl} \\
\text { solution }\end{array}$ & $\begin{array}{c}\text { Silver } \\
\text { nanoparticles from } \\
\text { Artemisia annua } \\
\text { and Sida acuta } \\
\text { leaves extract }\end{array}$ & $\begin{array}{c}\text { UV-visible } \\
\text { spectrophotometer }\end{array}$ & $\begin{array}{l}\text { Antifungal activity was studied } \\
\text { against Candida albicans. The } \\
\text { results of the antimicrobial } \\
\text { studies showed good inhibitory } \\
\text { effect against Staphylococcus } \\
\text { aureus, Escherichia coli and } \\
\text { Candida albican. Also, anti- } \\
\text { corrosion activity of these } \\
\text { silver nanoparticles on the } \\
\text { corrosion of mild steel in } \\
0.5 \text { M HCl solution was } \\
\text { studied by potentiodynamic } \\
\text { polarization curves and the } \\
\text { results show that they are good } \\
\text { inhibitors. The antioxidant } \\
\text { activity of both the extracts and } \\
\text { synthesized AgNP's was } \\
\text { analyzed by 1,1-diphenyl-2- } \\
\text { picryl-hydrazyl (DPPH) radical } \\
\text { scavenging assay using } \\
\text { ascorbic acid as control and } \\
\text { they were all found to exhibit } \\
\text { good antioxidant activity } \\
\text { especially at lower } \\
\text { concentrations. }\end{array}$ & 97 \\
\hline 98 & $\begin{array}{c}\text { Mild steel in } \\
1 \mathrm{M} \\
\text { hydrochloric } \\
\text { acid }\end{array}$ & $\begin{array}{l}\text { Salvia officinalis } \\
\text { essential oil }\end{array}$ & $\begin{array}{l}\text { Principal component } \\
\text { analysis (PCA), } \\
\text { weight loss } \\
\text { measurements, } \\
\text { potentiodynamic } \\
\text { polarization, }\end{array}$ & $\begin{array}{l}\text { Tafel polarization study } \\
\text { revealed that extract of } S \text {. } \\
\text { officinalis acts as a mixed type } \\
\text { inhibitor. Inhibition was found } \\
\text { to increase with increasing } \\
\text { concentration of the essential }\end{array}$ & 98 \\
\hline
\end{tabular}




\begin{tabular}{|c|c|c|c|c|c|}
\hline No. & $\begin{array}{l}\text { Metal / } \\
\text { medium }\end{array}$ & $\begin{array}{l}\text { Inhibitor / } \\
\text { Additives }\end{array}$ & Methods & Findings & Ref. \\
\hline & & & $\begin{array}{c}\text { electrochemical } \\
\text { impedance } \\
\text { spectroscopy (EIS) }\end{array}$ & $\begin{array}{l}\text { oil and extract of } S \text {. Officinalis. } \\
\text { Values of inhibition efficiency } \\
\text { calculated from weight loss, } \\
\text { Tafel polarization curves, and } \\
\text { EIS are in good agreement. } \\
\text { The effect of temperature on } \\
\text { the corrosion behaviour of mild } \\
\text { steel in } 1 \mathrm{M} \mathrm{HCl} \mathrm{with} \mathrm{addition} \\
\text { of essential oil and extract was } \\
\text { also studied and } \\
\text { thermodynamic parameters } \\
\text { were determined and } \\
\text { discussed. }\end{array}$ & \\
\hline 99 & $\begin{array}{l}\text { Aluminium } \\
\text { alloy in HCI }\end{array}$ & $\begin{array}{l}\text { Adsorption and } \\
\text { inhibitive } \\
\text { properties of } \\
\text { Camellia sinensis }\end{array}$ & $\begin{array}{c}\text { Weight } \\
\text { loss/corrosion rate } \\
\text { and potential } \\
\text { measurement } \\
\text { techniques }\end{array}$ & $\begin{array}{l}\text { The results obtained showed } \\
\text { effective corrosion inhibition } \\
\text { of the extract on the aluminium } \\
\text { alloy test specimens in the } \\
\text { different concentrations of } \\
\text { hydrochloric acid used. The } \\
\text { extracts gave appreciable } \\
\text { corrosion inhibition } \\
\text { performance of aluminium at } \\
100 \text { and } 80 \% \text { concentrations } \\
\text { with the weight loss of } 652 \text { mg } \\
\text { (0.652 g) and 674 mg (0.674 g) } \\
\text { respectively. ANOVA test } \\
\text { confirmed the results at } 95 \% \\
\text { confidence, and further showed } \\
\text { that concentration of green tea } \\
\text { extract had greater effect on } \\
\text { potential measurements } \\
\text { whereas test exposure time had } \\
\text { greater influence on weight } \\
\text { loss measurements. The value } \\
\text { of Gibb's free energy of } \\
\text { adsorption obtained signified } \\
\text { that the mechanism of } \\
\text { adsorption of plant extract } \\
\text { molecules on the metal surface } \\
\text { was by physiosorption. The } \\
\text { adsorption of Camellia sinensis } \\
\text { extract on the surface of } \\
\text { aluminium obeys the } \\
\text { Freundlich isotherm model. }\end{array}$ & 99 \\
\hline
\end{tabular}




\begin{tabular}{|c|c|c|c|c|c|}
\hline No. & $\begin{array}{l}\text { Metal / } \\
\text { medium }\end{array}$ & $\begin{array}{l}\text { Inhibitor / } \\
\text { Additives }\end{array}$ & Methods & Findings & Ref. \\
\hline 100 & $\begin{array}{l}\text { Mild steel in } \\
\text { acidic } \\
\text { environment }\end{array}$ & $\begin{array}{l}\text { Asteraceae } \\
\text { family- } \\
\text { Parthenium } \\
\text { hysterophorus } \\
\text { plant leaves } \\
\text { extract }\end{array}$ & $\begin{array}{c}\text { Weight loss } \\
\text { measurements, Tafel } \\
\text { polarization and } \\
\text { electrochemical } \\
\text { impedancespectrosc } \\
\text { opy techniques }\end{array}$ & $\begin{array}{l}\text { The maximum potential of the } \\
\text { extract was obtained at } \\
1100 \text { mg L-1 with } 84 \% \\
\text { efficiency. It was found that } \\
\text { adsorption of Parthenium } \\
\text { hysterophorus extract follows } \\
\text { Langmuir isotherm model. } \\
\text { Mechanism of corrosion } \\
\text { inhibition is explored with the } \\
\text { help of UV-vis spectra and } \\
\text { SEM images. }\end{array}$ & 100 \\
\hline
\end{tabular}

\section{Some studies in detail}

Rajendran et al. have done extensive work in the field of using green inhibitors [101-104].

\subsection{Corrosion inhibition of carbons steel in chloride medium by extract of Henna leaves}

An aqueous extract of leaves of Henna (Lawsonia inermis L.) powder has been used as a corrosion inhibitor in controlling corrosion of carbon steel immersed in an aqueous solution containing $60 \mathrm{ppm}$ of $\mathrm{Cl}^{-}$. Weight loss method has been used to calculate the inhibition efficiency. The experiments were carried out in the absence and presence of $\mathrm{Zn}^{2+}$. It is well known that the main constituent of this plant extract is Lawsone. It is observed that it has good inhibition efficiency (IE) and shows excellent IE at $\mathrm{pH} \mathrm{6,8}$ and 12. Interestingly in the presence of $\mathrm{Zn}^{2+}$ a synergistic effect is noticed. Synergism parameters have been calculated to evaluate the synergistic effect. Analysis of variance (Ftest) reveals that the synergistic effect existing between henna extract and $\mathrm{Zn}^{2+}$ in statistically significant. The protective film has been analyzed using Fourier transform infrared (FTIR) spectroscopy. The film consists of $\mathrm{Fe}^{2+}$-Lawsone complex and zinc hydroxide .It is found to be UV-fluorescent. Electro chemical studies such as potentiodynamic polarization and alternating current (AC) impedance have been used to find the mechanistic aspects of corrosion inhibition [101].

\subsection{Use of extracts of Henna leaves in prevention of concrete corrosion}

Green inhibitors have been used in concrete technology also. The corrosion resistance of mild steel in simulated concrete pore solution (SCPS) prepared in seawater has been investigated. Weight loss method, polarization study and AC impedance spectra have been used in this study. The influence of green inhibitor extracted from henna leaves has also been investigated in the absence and the presence of $\mathrm{Zn}^{2+}$ ions. It is noted that, in presence of this environmentally friendly green inhibitor (henna extract) the corrosion resistance of mild steel increases. This formulation may find application in concrete technology especially in 
marine environment because the experiments have been carried out in sea water. While constructing bridge in seas this findings may be taken into consideration [102].

\subsection{Inhibition of corrosion of carbon steel in sea water by extracts of Henna leaves}

The inhibition efficiency (IE) of an aqueous extract of henna leaves in controlling corrosion of carbon steel in seawater has been evaluated by weight-loss method. It is observed that the formulation consisting of $8 \mathrm{ml}$ of henna extract (HE) and $25 \mathrm{ppm}$ of $\mathrm{Zn}^{2+}$ has $94 \%$ inhibition efficiency in controlling corrosion of carbon steel in sea water. Polarization study reveals that $\mathrm{HE}$ and $\mathrm{Zn}^{2+}$ system functions as mixed type inhibitor. AC impedance spectra reveal that protective film is formed on the metal surface. The nature of the metal surface has been analysed by FTIR spectra, SEM, and AFM analysis [103].

\subsection{Inhibition of corrosion of mild steel in well water by an aqueous extract of Adhatoda} vasica leaves

The inhibition of corrosion of mild steel in well water by an aqueous extract of Adhatoda vasica leaves has been investigated. The findings are discussed in this sections.

Adhatoda vasica leaves were shade dried. $50 \mathrm{~g}$ of the dried leaves were boiled in double distilled water. The suspended impurities were removed by filtration. The solution was made up to $100 \mathrm{ml}$ and used in the present study as inhibitor solution.

\subsubsection{Weight loss method}

The inhibition efficiency of an aqueous extract of Adhatoda vasica leaves in controlling corrosion of mild steel in well water has been evaluated by weight loss method. The results are summarized in Table 6.

It is observed from the Table 6 that as the concentration of Adhatoda vasica leaves extract increases, the corrosion inhibition efficiency also increases. The active principle of Adhatoda vasica leaves extract, namely vasicine, vasicinone has coordinated with $\mathrm{Fe}^{2+}$ ions on the metal surface and forms a protective film consisting of $\mathrm{Fe}^{2+}$-vasicine and/or $\mathrm{Fe}^{2+}$ vasicinone complex. Thus the anodic reaction of metal dissolution is prevented. $92 \%$ inhibition is offered by $10 \mathrm{ml}$ of the extract.

Table 6: Corrosion inhibition efficiency of Adhatoda vasica leaves extract in controlling corrosion of mild steel in well water.

\begin{tabular}{ccc}
\hline $\begin{array}{c}\text { Volume of Adhatoda vasica } \\
\text { leaves extract, } \mathbf{~ m L}\end{array}$ & Corrosion rate (CR), mdd & IE \% \\
\hline 0 & 32.68 & - \\
2 & 17.64 & 46 \\
4 & 14.05 & 57 \\
6 & 9.80 & 70 \\
8 & 6.2 & 81 \\
10 & 2.61 & 92 \\
\hline
\end{tabular}




\subsection{Polarization study}

Polarization study is useful in confirming the formation of protective film formed on the metal surface. If a protective film is formed, the linear polarization resistance increases and the corrosion current value decreases. The polarization curves of mild steel immersed in various test solutions are shown in Figure 1.

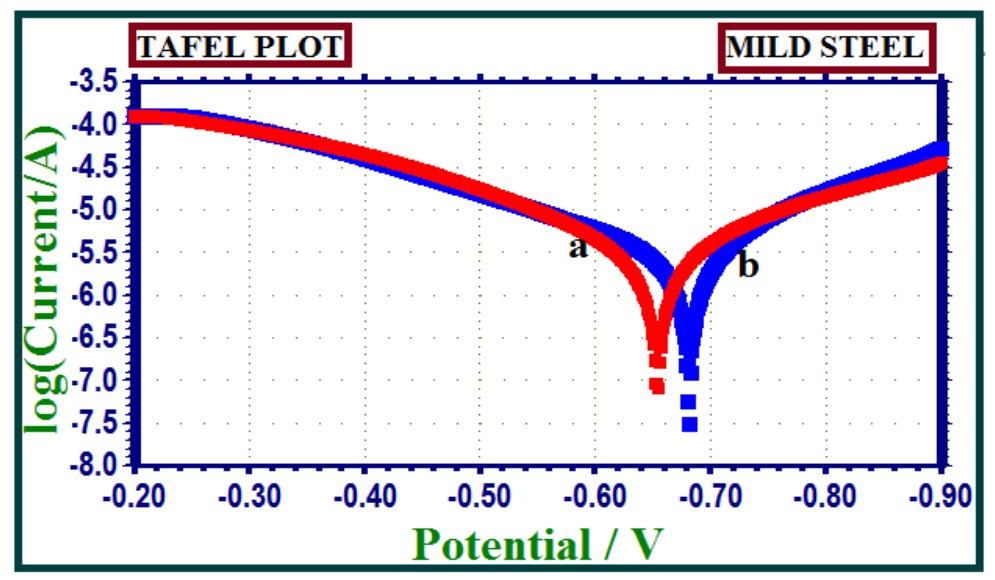

Figure 1. Polarization curves of mild steel immersed in various test solutions. (a) well water, (b) well water $+10 \mathrm{ml}$ of Adhatoda vasica leaves extract.

The corrosion parameters such as corrosion potential $\left(E_{\text {corr }}\right)$, corrosion current $\left(I_{\text {corr }}\right)$, linear polarization resistance (LPR) and Tafel slopes $\left(b_{\mathrm{c}}=\right.$ cathodic; $b_{\mathrm{a}}=$ anodic $)$ are given in Table 7.

It is observed from Table 7, that when mild steel is immersed in well water, the corrosion potential is $-654 \mathrm{mV}$ vs. SCE. The corrosion current is $9.447 \cdot 10^{-6} \mathrm{~A} / \mathrm{cm}^{2}$. The LPR value is $4022.1 \mathrm{Ohm} \cdot \mathrm{cm}^{2}$. In the presence of inhibitor, the corrosion potential is shifted from -654 to $-682 \mathrm{mV} v s$. SCE. This is a cathodic shift. It suggests that the cathodic reaction is controlled predominantly. The shift is only in small range. Hence anodic and cathodic reactions are controlled to an equal extent. The LPR value increases from 4022.1 to $11108.3 \mathrm{Ohm} \cdot \mathrm{cm}^{2}$. The corrosion current decreases from $9.447 \cdot 10^{-6}$ to $3.61 \cdot 10^{-6} \mathrm{~A} / \mathrm{cm}^{2}$. These observations confirm that a protective film is formed on the metal surface. This controls the corrosion of metal.

Table 7. Corrosion parameters of mild steel immersed in well water in the absence and presence of an aqueous extract of Adhatoda vasica leaves (Polarization study)

\begin{tabular}{|c|c|c|c|c|c|c|}
\hline No. & System & $\begin{array}{r}E_{\text {corr }} \mathbf{m V} \\
v s . S C E\end{array}$ & $I_{\text {corr }} \mathrm{A} / \mathrm{cm}^{2}$ & $\begin{array}{c}\text { LPR } \\
\text { Ohm cm } \text { cm }^{2}\end{array}$ & $\begin{array}{c}b_{\mathrm{c}} \\
\mathrm{mV} / \mathrm{decade}\end{array}$ & $\begin{array}{c}b_{\mathrm{a}} \\
\mathrm{mV} / \text { decade }\end{array}$ \\
\hline 1 & Well water & -654 & $9.447 \cdot 10^{-6}$ & 4022.1 & 181.6 & 168.4 \\
\hline 2 & $\begin{array}{c}\text { Well water + Adhatoda } \\
\text { leaves extract }\end{array}$ & -682 & $3.61 \cdot 10^{-6}$ & 11108.3 & 155.9 & 225.8 \\
\hline
\end{tabular}




\subsubsection{AC impedance spectra}

AC impedance spectra are useful in confirming the formation of protective film formed on the metal surface. If a protective film is formed on the metal surface, the charge transfer resistance $\left(R_{\mathrm{t}}\right)$ value increases; double layer capacitance value $\left(C_{\mathrm{dl}}\right)$ decreases and the impedance $[\log (\mathrm{Z} / \mathrm{Ohm})]$ value increases. The AC impedance spectra of mild steel immersed in well water in presence of inhibitor system are shown in the Figure 2 and Figure 3. The Nyquist plots are shown in the Figure 2. The Bode plots are shown in Figure $3 \mathrm{a}$ and Figure $3 \mathrm{~b}$. The corrosion parameters, namely, $R_{\mathrm{t}}, C_{\mathrm{dl}}$ and impedance values are given in Table 8.

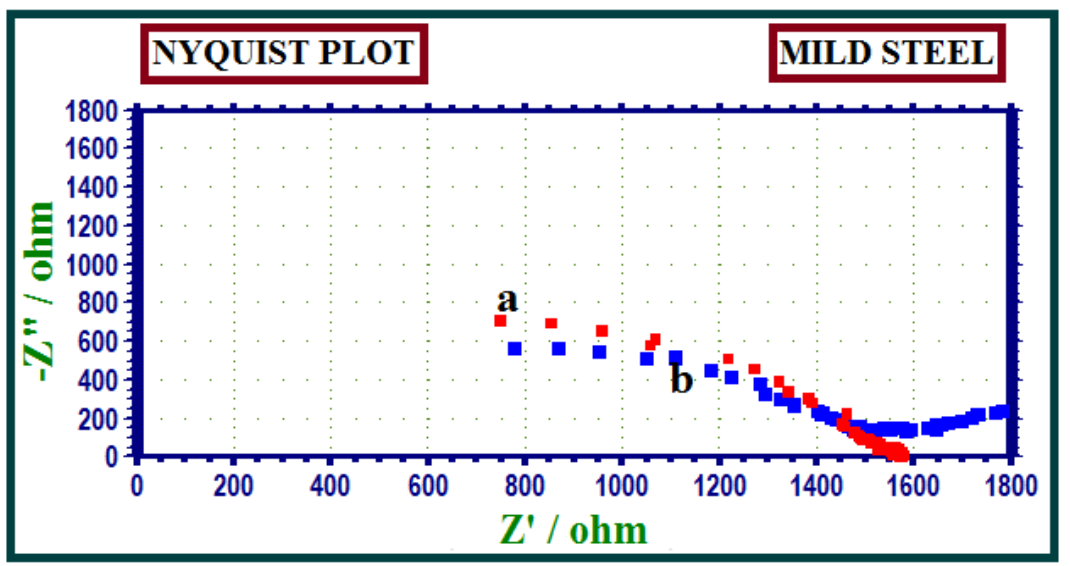

Figure 2. AC impedance spectra of mild steel immersed in various test solution (Nyquist Plot) (a) Well water (b) Well water $+10 \mathrm{~mL}$ of Adhatoda vasica leaves extract.

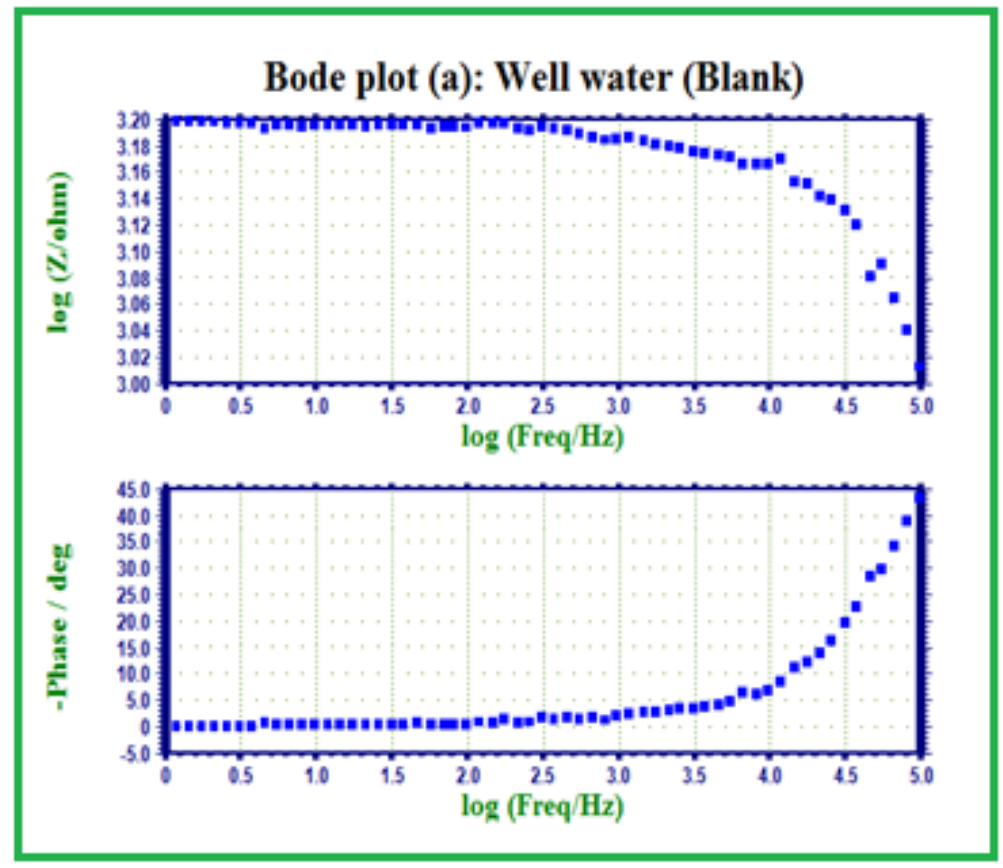

Figure 3a. AC impedance spectra of mild steel immersed in well water (Bode Plot). 


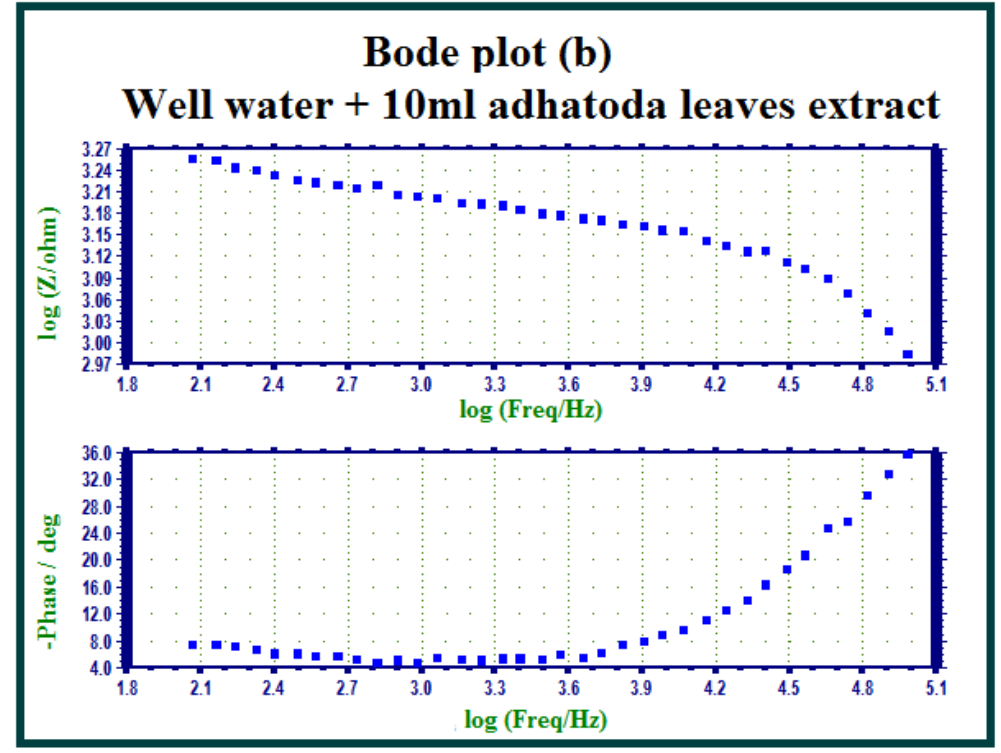

Figure 3b. AC impedance spectra of mild steel immersed in well water $+10 \mathrm{~mL}$ of Adhatoda vasica leaves extract (Bode Plot).

It is observed from Table 8 that when mild steel is immersed in well water, the charge transfer resistance is $286.7 \mathrm{Ohm} \mathrm{cm}$. The double layer capacitance is $17.739 \cdot 10^{-9} \mathrm{~F} \mathrm{~cm}^{-2}$. The impedance value is 2.721 . In the presence of inhibitor $(10 \mathrm{ml}$ of Adhatoda vasica leaves extract), the charge transfer resistance value $\left(R_{\mathrm{t}}\right)$ increases from $286.7 \mathrm{Ohm} \mathrm{cm}^{2}$ to $921 \mathrm{Ohm} \mathrm{cm} \mathrm{cm}^{2}$. The double layer capacitance value $\left(C_{\mathrm{dl}}\right)$ decreases from $17.739 \cdot 10^{-9} \mathrm{~F} \mathrm{~cm}^{-2}$ to $5.428 \cdot 10^{-9} \mathrm{~F} \mathrm{~cm}^{-2}$. The impedance $\log$ (Z/Ohm) increases from 2.721 to 3.201.

Table 8. Corrosion parameters of mild steel immersed in well water in presence of adhatoda vasica leaves extract obtained from AC impedance spectra.

\begin{tabular}{cccc}
\hline & \multicolumn{2}{c}{ Nyquist plot } & Bode plot \\
\cline { 2 - 4 } System & $\begin{array}{c}\boldsymbol{R}_{\mathbf{t}} \\
\text { Ohm cm }\end{array}$ & $\begin{array}{c}\boldsymbol{C}_{\mathbf{d l}} \\
\mathbf{F ~ c m ~}^{-2}\end{array}$ & Impedance log (Z/ohm) \\
\hline Well water (blank) & 286.7 & $17.739 \cdot 10^{-9}$ & 2.721 \\
$\begin{array}{c}\text { Well water + 10 ml } \\
\text { of Adhatoda vasica } \\
\text { leaves extract }\end{array}$ & 921 & $5.428 \cdot 10^{-9}$ & 3.201 \\
\hline
\end{tabular}

These observations confirm that a protective film is formed on the metal surface. This prevents the transfer of electrons from the metal to the solution medium. Thus corrosion of mild steel is prevented. 


\subsubsection{AFM (Atomic Force Microscopy) studies}

The 2D AFM images of polished metal surface, corroded surface (immersed in well water) and the film protected metal (well water +inhibitor solution) are shown in Figure 4a, Figure $4 \mathrm{~b}$ and Figure $4 \mathrm{c}$. The corresponding 3D images are shown in Figure 5a, Figure $5 \mathrm{~b}$ and Figure 5c. The AFM parameters RMS $\left(R_{\mathrm{q}}\right)$ roughness $(\mathrm{nm})$, Average $\left(R_{\mathrm{a}}\right)$ roughness $(\mathrm{nm})$ and maximum peak-to-valley height $(\mathrm{nm})$ were calculated. These values are given in Table 9 for polished mild steel, mild steel immersed in well water and mild steel immersed in inhibitor system.

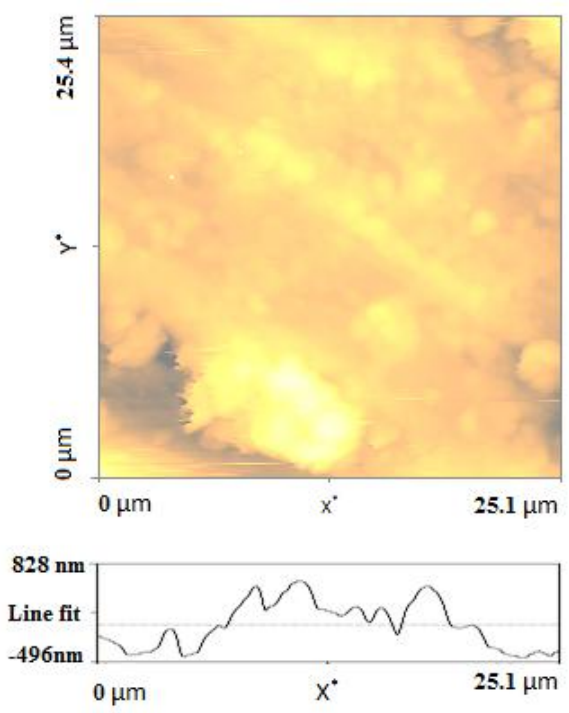

(a)
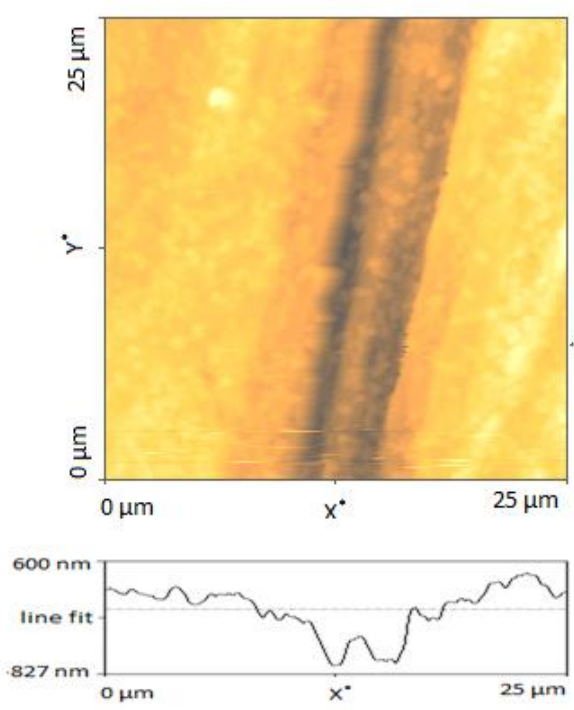

(b)

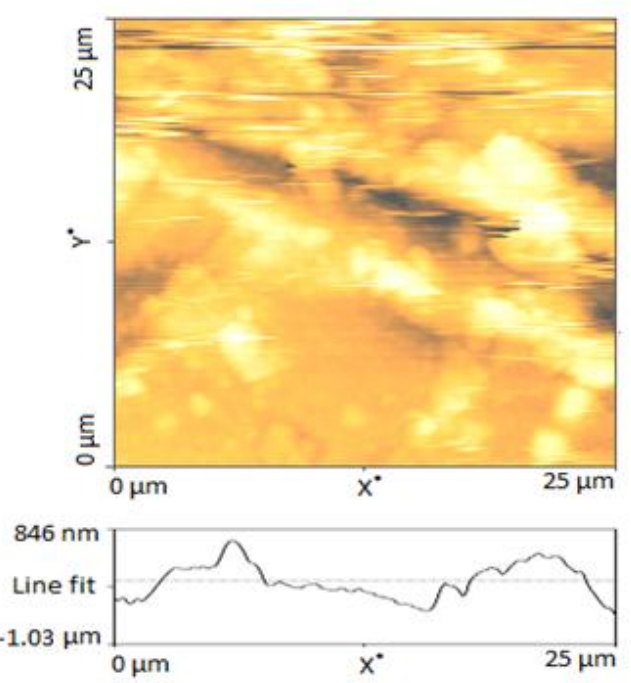

(c)

Figure 4. 2D AFM images of surface (a) polished metal, (b) mild steel immersed in well water, (c) mild steel immersed in well water containing adhatoda vasica leaves extract. 
Table 8. AFM parameters of mild steel surface in the presence and absence of inhibitor (Adhatoda vasica leaves system).

\begin{tabular}{cccc}
\hline Sample & $\begin{array}{c}\text { RMS }\left(\boldsymbol{R}_{\mathbf{q}}\right) \\
\text { Roughness }(\mathbf{n m})\end{array}$ & $\begin{array}{c}\text { Average }\left(\boldsymbol{R}_{\mathbf{a}}\right) \\
\text { Roughness }(\mathbf{n m})\end{array}$ & $\begin{array}{c}\text { Maximum peak-to- } \\
\text { valley height }(\mathbf{n m})\end{array}$ \\
\hline Polished metal & 135.9 & 172.84 & 806.9 \\
\hline $\begin{array}{c}\text { Mild steel immersed in } \\
\text { well water (Blank) }\end{array}$ & 513.53 & 637.27 & 2885.3 \\
\hline $\begin{array}{c}\text { Mild steel immersed in } \\
\text { well water and 10 mL } \\
\text { of Adhatoda vasica } \\
\text { leaves extract }\end{array}$ & 203.86 & 265.82 & 1152.6 \\
\hline
\end{tabular}

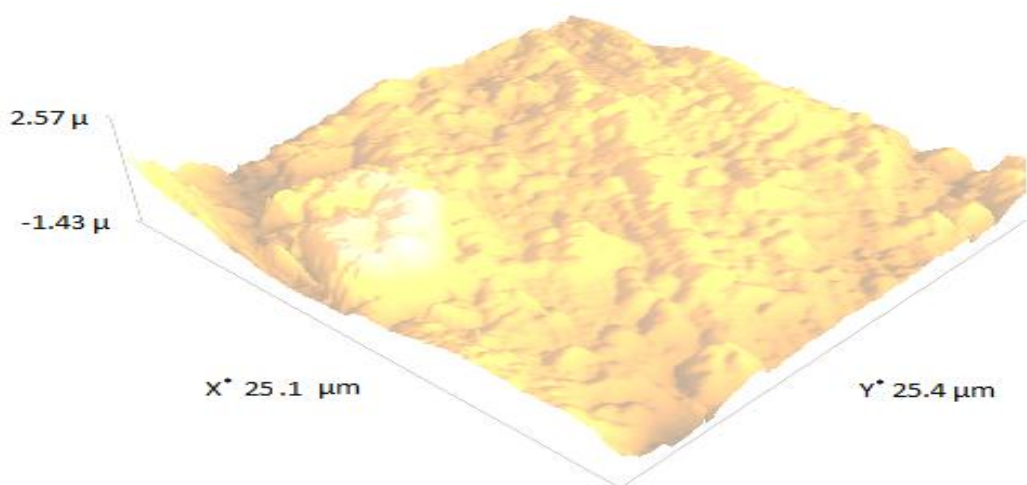

(a)

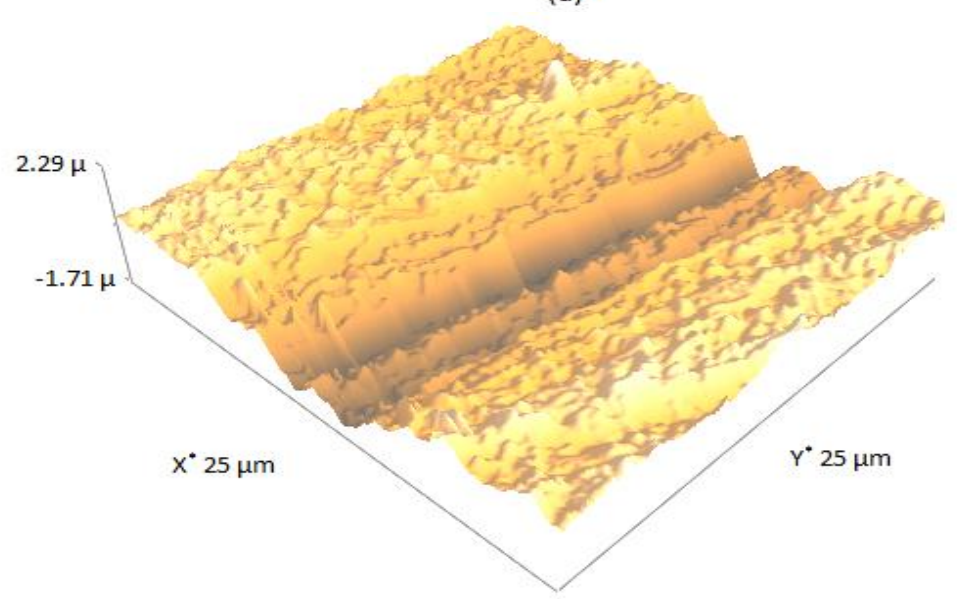

(b) 


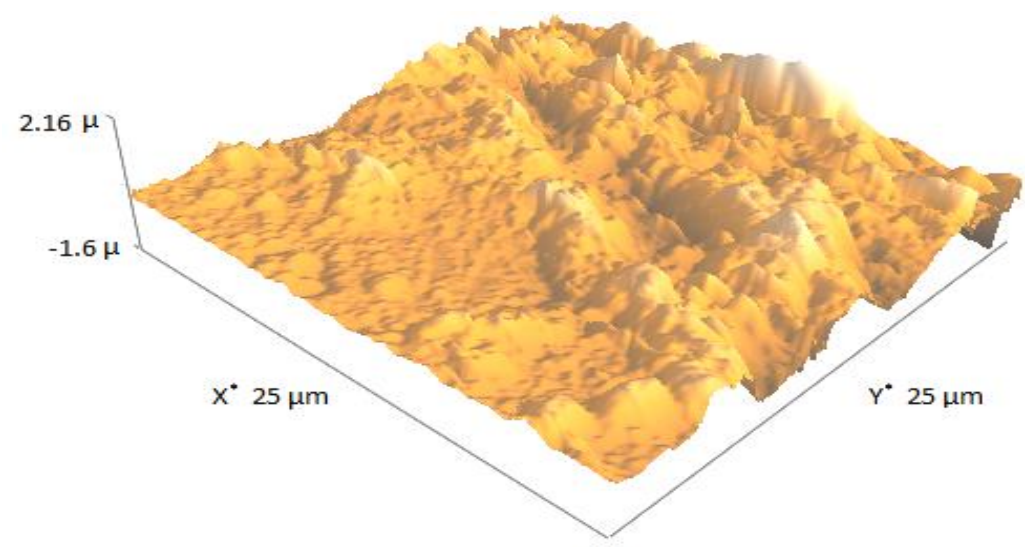

(c)

Figure 5. 3D AFM images of surface (a) polished metal, (b) mild steel immersed in well water, (c) mild steel immersed in well water containing adhatoda vasica leaves extract

It is observed from the Table 8 that the RMS roughness of polished mild steel is $135.9 \mathrm{~nm}$. The average roughness is $172.84 \mathrm{~nm}$. The maximum peak-to-valley height is $806.9 \mathrm{~nm}$. Analysis of Table 8 reveals that the RMS roughness value of mild steel immersed in well water is higher than that of the polished mild steel. This indicates that due to corrosion, thick nanofilm is formed on the metal surface. Similar is in the case of average roughness and maximum peak-to-valley height. For the metal immersed in inhibitor system, the RMS roughness is higher than that of the polished metal, but lower than that of the metal immersed in corrosive medium. Similar is the case in the case of average roughness and maximum peak-to-valley height. This indicates that a protective film of nano scale is formed on the metal surface. This film protects the metal from corrosion.

Thus the above section explains how weight loss method, electrochemical studies and AFM spectra are used in green inhibitor study.

\section{Acknowledgment}

Writing a review report is not an easy work. It is a difficult one. However this work has been done easily with the help of our Young and Energetic Scientific Team. I had the feeling of walking gently in a flowering garden, taking the gentle hands of the breeze. They readily helped us by allotting time for this work amidst their regular work. The authors are thankful to Prof. S. Christina Joycee and Prof. S. Gowri, both from St. Antony's College, Dindigul, Prof. S. Devinmeenakshi from NS College, Theni, Prof. S. Syed Abuthaheer, Jamal Mohamed College, Trichy and Prof. N. Karthiga, SBM College of Engineering and Technology, Dindigul. 


\section{References}

1. M. Benarioua, A. Mihi, N. Bouzeghaia and M. Naoun, Mild steel corrosion inhibition by Parsley (Petroselium Sativum) extract in acidic media, Egypt. J. Pet., 2019 (in press).

2. M. Ramezanzadeh, G. Bahlakeh, Z. Sanaei and B. Ramezanzadeh, Studying the Urtica dioica leaves extract inhibition effect on the mild steel corrosion in $1 \mathrm{M} \mathrm{HCl}$ solution: Complementary experimental, ab initio quantum mechanics, Monte Carlo and molecular dynamics studies, J. Mol. Liq., 2018, 272, 120-136.

3. O. Olawale, B.T. Ogunsemi J.O. Bello, P.P. Ikubanni, S.J. Ogundipe and T.S. Abayomi, Optimisation and modelling of aluminium corrosion inhibition using almond (Prunus amydgdalus) fruit leaves extract as green inhibitor in $\mathrm{HCl}$ acidic medium, Int. J. Mech. Eng. Technol., 2018, 9, no. 13, 1274-1285.

4. Z. Jamshidnejad, A. Afshar and M.A. Razmjoo Khollari, Synthesis of self-healing smart epoxy and polyurethane coating by encapsulation of olive leaf extract as corrosion inhibitor, Int. J. Electrochem. Sci., 2018, 13, no. 12, 12278-12293.

5. O. Olawale, B.T. Ogunsemi, O.O. Agboola, M.B. Ake and G.O. Jawando, Inhibition effect of orange seed extract on aluminium corrosion in $1 \mathrm{M}$ hydrochloric acid solution, Int. J. Mech. Eng. Technol., 2018, 9, 282-287.

6. M.A. Asaad, M. Ismail, M.M. Tahir, G.F. Huseien, P.B. Raja and Y.P. Asmara, Enhanced corrosion resistance of reinforced concrete: Role of emerging eco-friendly Elaeis guineensis/silver nanoparticles inhibitor, Constr. Build. Mater., 2018, 188, 555568.

7. J.C. Pritchard, Y.-M. Cho, N. Ashoori, J.M. Wolfand, J.D. Sutton, M.E. Carolan, E. Gamez, K. Doan, J.S. Wiley and R.G. Luthy, Benzotriazole uptake and removal in vegetated biofilter mesocosms planted with Carex praegracilis, Water, 2018, 10, no. 11, 1605. doi: 10.3390/w10111605

8. S. Abrishami, R. Naderi and B. Ramezanzadeh, Fabrication and characterization of zinc acetylacetonate/Urtica Dioica leaves extract complex as an effective organic/inorganic hybrid corrosion inhibitive pigment for mild steel protection in chloride solution, Appl. Surf. Sci., 2018, 457, 487-496.

9. M.A. Asaad, M. Ismail, N.H.A. Khalid, G.F. Huseien and P.B. Raja, Elaeis guineensis leaves extracts as eco-friendly corrosion inhibitor for mild steel in hydrochloric acid, Jurnal Teknologi, 2018, 80, no. 6, 53-59.

10. V. Sribharathy, K. Kavipriya, N. Vellachi and K. Vijaya, Azadirachta indica L.extracts as green inhibitor for mild steel in sea water, Int. J. Mech. Eng. Technol., 2018, 9, no. $11,1614-1621$.

11. A. Berrani, H. Benassaoui, M. Zouarhi, A. Alrhorfi, N. Hajjaji and R. Bengueddour, Evaluation of inhibitory effect of the methanolic extract of the two parts from anabasis aretioides plant against the corrosion of $\mathrm{E} 24$ steel in a neutral solution $\mathrm{NaCl} 3.5 \%$, Anal. and Bioanal. Electrochem., 2018, 10, no 10, 1299-1316. 
12. A.N.F. Ganda, P.H. Andoko Setyarini and F. Gapsari, The inhibitive effect of tannin in Psidium guajava leaves towards $304 \mathrm{SS}$ corrosion in concentrated $\mathrm{HCl}$, MATEC Web of Conferences Open Access, 2018, 204, 05018.

13. C. Verma, E.E. Ebenso, I. Bahadur and M.A. Quraishi, An overview on plant extracts as environmental sustainable and green corrosion inhibitors for metals and alloys in aggressive corrosive media, J. Mol. Liq., 2018, 266, 577-590.

14. A.S. Fouda and E. Abdel Haleem, Berry Leaves Extract as Green Effective Corrosion Inhibitor for $\mathrm{Cu}$ in Nitric Acid Solutions, Surf. Eng. Appl. Electrochem., 2018, 54, no. 5, 498-507.

15. C. Verma, M.A. Quraishi, E.E. Ebenso and I. Bahadur, A Green and Sustainable Approach for Mild Steel Acidic Corrosion Inhibition Using Leaves Extract: Experimental and DFT Studies, J. Bio Tribo Corros., 2018, 4, no. 3, 33.

16. Y. Lekbach, D. Xu, S. El Abed, Y. Dong, D. Liu, M. Saleem Khan, S. Ibnsouda Koraichi and K. Yang, Mitigation of microbiologically influenced corrosion of 304L stainless steel in the presence of Pseudomonas aeruginosa by Cistus ladanifer leaves extract, Int. Biodeterior. Biodegrad., 2018, 133, 159-169. doi: 10.1016/j.ibiod.2018.07.003

17. T.K. Bhuvaneswari, V.S. Vasantha and C. Jeyaprabha, Pongamia Pinnata as a Green Corrosion Inhibitor for Mild Steel in 1N Sulfuric Acid Medium, Silicon, 2018, 10, no. $5,1793-1807$.

18. D.K. Verma, F. Khan, I. Bahadur, M. Salman, M.A. Quraishi, C. Verma and E.E. Ebenso, Inhibition performance of Glycine max, Cuscuta reflexa and Spirogyra extracts for mild steel dissolution in acidic medium: Density functional theory and experimental studies, Results Phys., 2018, 10, September, 665-674.

19. M. Ali Asaad, N.N. Sarbini, A. Sulaiman, M. Ismail, G.F. Huseien, Z. Abdul Majid and P. Bothi Raja, Improved corrosion resistance of mild steel against acid activation: Impact of novel Elaeis guineensis and silver nanoparticles, J. Ind. Eng. Chem., 2018, 63, 139-148.

20. A.S Ogunbadejo, S. Aribo, O.A. Olaseinde, O.O. Ige and P. Olubambi, Correlation between repassivation kinetics and pitting potential of 316L stainless steel in the presence of green inhibitors, Pigm. Resin Technol., 2018, 47, no. 4, 338-349.

21. AA. Khadom, A.N. Abd and N.A. Ahmed, Xanthium strumarium leaves extracts as a friendly corrosion inhibitor of low carbon steel in hydrochloric acid: Kinetics and mathematical studies, S. Afr. J. Chem. Eng., 2018, 25, 13-21.

22. S.O. Anuchi and N.C. Ngobiri, Corrosion inhibition of mild steel in a $\mathrm{H}_{2} \mathrm{SO}_{4}$ solution by piper guineense squeezed extract, Port. Electrochim. Acta, 2018, 36, no. 4, 285-291.

23. O. Sanumi and E. Makhatha, Corrosion behavior of synthetic polymer inhibitors in acidic environment: A review, IEEE 9th International Conference on Mechanical and Intelligent Manufacturing Technologies, ICMIMT 2018 2018-January, 69-73. 
24. W.M.K.W.M. Ikhmal, M.Y.N. Yasmin, M.F.M. Fazira, W. A. W. Rafizah, W.B. Wan Nik and M.G.M. Sabri, Anticorrosion Coating using Olea sp. Leaves Extract, IOP Conf. Ser.: Mater. Sci. Eng., 2018, 344, no. 1, 012028.

25. M. Ramezanzadeh, Z. Sanaei, G. Bahlakeh and B. Ramezanzadeh, Highly effective inhibition of mild steel corrosion in $3.5 \% \mathrm{NaCl}$ solution by green Nettle leaves extract and synergistic effect of eco-friendly cerium nitrate additive: Experimental, MD simulation and QM investigations, J. Mol. Liq., 2018, 256, 67-83.

26. E. Alibakhshi, M. Ramezanzadeh, G. Bahlakeh, B. Ramezanzadeh, M. Mahdavian and M. Motamedi, Glycyrrhiza glabra leaves extract as a green corrosion inhibitor for mild steel in $1 \mathrm{M}$ hydrochloric acid solution: Experimental, molecular dynamics, Monte Carlo and quantum mechanics study, J. Mol. Liq., 2018, 255, 185-198.

27. A.A. Ganash, Theoretical and experimental studies of dried marjoram leaves extract as green inhibitor for corrosion protection of steel substrate in acidic solution, Chem. Eng. Commun., 2018, 205, no. 3, 350-362.

28. X. Gu, K. Dong, J. Tian, H. Li, J. Zhang, C. Qu and G. Chen, Investigation of modified ginkgo biloba leaves extract as eco-friendly inhibitor for the corrosion of N80 steel in 5\% HCL, Desalin. Water Treat., 2018, 107, 118-126.

29. A. Rodríguez-Torres, O. Olivares-Xometl, M.G. Valladares-Cisneros and J.G. González-Rodríguez, Effect of green corrosion inhibition by Prunus persica on AISI 1018 carbon steel in $0.5 \mathrm{M} \mathrm{H}_{2} \mathrm{SO}_{4}$, Int. J. Electrochem. Sci., 2018, 13, no. 3, 3023-3049.

30. M. Idrees, S. Batool, T. Kalsoom, S. Raina, H.M.A. Sharif and S. Yasmeen, Biosynthesis of silver nanoparticles using Sida acuta extract for antimicrobial actions and corrosion inhibition potential, Environ. Technol. (UK), 2019, 40, 1071-1078. doi: $\underline{10.1080 / 09593330.2018 .1435738}$

31. A.B. Hamdan, F.I Suryanto and Haider, Study on tea leaves extract as green corrosion inhibitor of mild steel in hydrochloric acid solution, IOP Conf. Ser.: Mater. Sci. Eng., 2018, 290, no. 1, 012086.

32. K. Yadav, A. Gupta, S.N. Victoria and R. Manivannan, Murraya koenigii as Green corrosion inhibitor for mild steel in nitric acid medium, Indian J. Chem. Technol., 2018, 25, no. 1, 94-100.

33. A.A. Khadom and B.M. Abod, Ziziphus leaves extract as friendly inhibitor for galvanic corrosion of steel-copper couple in petroleum waste water, ARPN J. Eng. Appl. Sci., 2018, 13, no. 4, 861-866.

34. M.A. Benghalia, C. Fares, A. Khadraoui, M. HadjMeliani, I.B. Obot, A. Sorrour, M. Dmytrakh and Z. Azari, Performance evaluation of a natural and synthetic compound as corrosion inhibitors of API 51 X52 steel in hydrochloric acid media, Moroccan J. Chem., 2018, 6, no. 1, 51-61.

35. H. Kumar and V. Yadav, Asian J. Chem., Aloe vera L. as green corrosion inhibitor for mild steel in 5.0 M hydrochloric acid solution, 2018, 30, no. 3, 474-478. 
36. A.I. Ikeuba and P.C. Okafor, Green corrosion protection for mild steel in acidic media: saponins and crude extracts of Gongronema latifolium, Pigm. Resin Technol., 2018, 48, no. $1,57$.

37. R. Prasanna, P. Manonmani, R. Elangomathavan and M. Goel, Anti corrosion studies on ethanolic extract of Hibiscus Rosa sinensis and azadirachita Indica leaves, Int. J. Civil Eng. Technol., 2018, 9, no. 1, 219-229.

38. N. Raghavendra and J.I. Bhat, Anti-corrosion properties of areca palm leaf extract on aluminium in $0.5 \mathrm{M} \mathrm{HCl}$ environment, S. Afr. J. Chem., 2018, 71, 30-38.

39. I.-M. Chung, V. Hemapriya, K. Ponnusamy, N. Arunadevi, S. Chitra, H.-Y. Chi, S.H. Kim and M. Prabakaran, Assessment of low carbon steel corrosion inhibition by eco-friendly green chaenomeles sinensis extract in acid medium, J. Electrochem. Sci. Technol., 2018, 9, no. 3, 238-249.

40. T.H. Ibrahim, E.E. Gomes, I.B. Obot, M. Khamis and M.A. Sabri, Mild steel green inhibition by Ficus carica leaves extract under practical field conditions, J. Adhes. Sci. Technol., 2017, 31, no. 24, 2697-2718.

41. Y.P. Asmara, V. Suraj, J.P. Siregar, T. Kurniawan, D. Bachtiar and N.M.Z.N. Mohamed, Development of green vapour corrosion inhibitor, IOP Conf. Ser.: Mater. Sci. Eng., 2017, 257, no. 1, 012089.

42. M.A. Asaad, M. Ismail, P.B. Raja and N.H.A. Khalid, Rhizophora Apiculata As EcoFriendly Inhibitor Against Mild Steel Corrosion in $1 \mathrm{M} \mathrm{HCl,} \mathrm{Surf.} \mathrm{Rev.} \mathrm{Lett.,} \mathrm{2017,} \mathrm{24,}$ 1850013.

43. B. Ramezanzadeh, P. Kardar, G. Bahlakeh, Y. Hayatgheib and M. Mahdavian, Fabrication of a Highly Tunable Graphene Oxide Composite through Layer-by-Layer Assembly of Highly Crystalline Polyaniline Nanofibers and Green Corrosion Inhibitors: Complementary Experimental and First-Principles Quantum-Mechanics Modeling Approaches, J. Phys. Chem. C, 2017, 121, no 37, 20433-20450.

44. S.A. Rao and P. Rao, Corrosion inhibition and adsorption behavior of Murraya koenigii extract for corrosion control of aluminum in hydrochloric acid medium, Surf. Eng. Appl. Electrochem., 2017, 53, no. 5, 475-485.

45. S. Kadapparambil, K. Yadav, M. Ramachandran and N. Victoria Selvam, Electrochemical investigation of the corrosion inhibition mechanism of Tectona grandis leaf extract for SS304 stainless steel in hydrochloric acid, Corros. Rev., 2017, 35, no. 2, 111-121.

46. V. Koundal, R. Haldhar, A. Saxena and D. Prasad, Natural non poisonous green inhibitor of Glycyrrhiza glabra for mild steel in 3.5\% NaCl, AIP Conf. Proc., 2017, 1860, 020063.

47. S. Perumal, S. Muthumanickam, A. Elangovan, R. Karthik, R.S. Kannan and K.K. Mothilal, Bauhinia tomentosa Leaves Extract as Green Corrosion Inhibitor for Mild Steel in 1M HCl Medium, J. Bio Tribo Corros., 2017, 3, no. 2, 13. 
48. R.S. Nathiya and V. Raj, Evaluation of Dryopteris cochleata leaf extracts as green inhibitor for corrosion of aluminium in $1 \mathrm{M} \mathrm{H}_{2} \mathrm{SO}_{4}$, Egypt. J. Pet., 2017, 26, no. 2, 313-323.

49. A. Ehsani, M.G. Mahjani, M. Hosseini, R. Safari, R. Moshrefi and H. Mohammad Shiri, Evaluation of Thymus vulgaris plant extract as an eco-friendly corrosion inhibitor for stainless steel 304 in acidic solution by means of electrochemical impedance spectroscopy, electrochemical noise analysis and density functional theory, J. Colloid Interface Sci., 2017, 490, 444-451.

50. A. Djouahri, S. Sebiane, F. Kellou, L. Lamari, N. Sabaou, A. Baaliouamer and L. Boudarene, Inhibitory effect on corrosion of carbon steel in acidic media, antioxidant, antimicrobial, anti-5-lipooxygenase and anti-xanthine oxidase activities of essential oil from Tetraclinis articulata (Vahl) Masters leaves, J. Essent. Oil Res., 2017, 29, no. 2, 169-178.

51. S. Kherraf, E. Zouaoui and M.S. Medjram, Corrosion inhibition of Monel 400 in hydrochloric solution by some green leaves, Anti-Corros. Methods Mater., 2017, 64, no. 3, 347-354.

52. H. Bendaif, A. Melhaoui, A. Bouyanzer, B. Hammouti and Y. El Ouadi, The study of the aqueous extract of leaves of Pancratium Foetidum Pom as: Characterization of polyphenols, flavonoids, antioxidant activities and ecofriendly corrosion inhibitor, $J$. Mater. Environ. Sci., 2017, 8, no. 12, 4475-4486.

53. P.R. Sivakumar and A.P. Srikanth, Anticorrosive activity of Schreabera swietenioids leaves as green inhibitor for mild steel in acidic solution, Asian J. Chem., 2017, 29, no. 2, 274-278.

54. N.I. Prajapati and R.T. Vashi, Inhibitory effects of Delonix regia(Gulmohor) extract on corrosion of aluminium in hydrochloric acid, J. Corros. Sci. Eng., 2017.

55. S. Chen, A. Singh, Y. Wang, W. Liu, K. Deng and Y. Lin, Inhibition effect of Ilex kudingcha C.J. Tseng (Kudingcha) extract on J55 steel in $3.5 \mathrm{wt} \% \mathrm{NaCl}$ solution saturated with $\mathrm{CO}_{2}$, Int. J. Electrochem. Sci., 2017, 12, no. 1, 782-796.

56. J.K. Odusote and O.M. Ajayi, Iran. J. Mater. Sci. Eng., Corrosion inhibitive properties of extract of Jatropha Curcas leaves on mild steel in hydrochloric acid environment, 2016, 13, no. 3, 1-11.

57. S. Dahiya, S. Lata, P. Kumar and R. Kumar, A descriptive study for corrosion control of low-alloy steel by Aloe vera extract in acidic medium, Corros. Rev., 2016, 34, no. 4, 241-248.

58. A. Fattah-Alhosseini and B. Hamrahi, Effect of Thyme leaves hydroalcoholic extract on corrosion behavior of API 5L carbon steel in $0.5 \mathrm{M} \mathrm{H}_{2} \mathrm{SO}_{4}$, Anal. Bioanal. Electrochem., 2016, 8, no. 5, 535-546.

59. B.S. Swaroop, S.N. Victoria and R. Manivannan, Azadirachta indica leaves extract as inhibitor for microbial corrosion of copper by Arthrobacter sulfureus in neutral $\mathrm{pH}$ conditions-A remedy to blue green water problem, J. Taiwan Inst. Chem. Eng., 2016, 64, 269-278. 
60. M.S. Uwineza, M. Essahli and A. Lamiri, Corrosion inhibition of aluminium in $2 \mathrm{M}$ phosphoric acid using the essential oil of mentha pulegium leaves, Port. Electrochim. Acta, 2016, 34, no. 4, 53-62.

61. M. Jokar, T.S. Farahani and B. Ramezanzadeh, Electrochemical and surface characterizations of morus alba pendula leaves extract (MAPLE) as a green corrosion inhibitor for steel in 1M HCl, J. Taiwan Inst. Chem. Eng., 2016, 63, 436-452.

62. K. Hema, B.R. Venkatraman and A. Subramania, Potential of Ziziphus jujuba leaves extract as green corrosion inhibitor against carbon steel in $1 \mathrm{~N} \mathrm{HCl}$ solution, Pharma Chem., 2016, 8, no. 2, 332-342.

63. G.M. AL-Senani, S.I. AL-Saeedi and R.S. AL-Mufarij, Coriandrum sativum leaves extract (CSL) as an eco-friendly green inhibitor for corrosion of carbon steel in acidic media, Coriandrum sativum leaves extract (CSL) as an eco-friendly green inhibitor for corrosion of carbon steel in acidic media, Journal of Materials and Environmental Science, J. Mater. Environ. Sci., 2016, 7, no. 7, 2240-2251.

64. K. Vishalakshi, P.R. Sivakumar and A.P. Srikanth, Analysis of corrosion resistance behavior of green inhibitors on mild steel in $1 \mathrm{~N} \mathrm{HCl}$ medium using electrochemical techniques, Pharma Chem., 2016, 8, no. 19, 548-553.

65. K.K. Patel and R.T. Vashi, J. Corros. Sci. Eng., Lawsonia extract as a green corrosion inhibitor for Copper in Nitric Acid solution, Journal of Corrosion Science and Engineering, 2016, 19.

66. M. Prabakaran, S.-H. Kim, V. Hemapriya, M. Gopiraman, I.S. Kim and I.-M. Chung, Rhus verniciflua as a green corrosion inhibitor for mild steel in $1 \mathrm{M} \mathrm{H}_{2} \mathrm{SO}_{4}, R S C A d v$., 6, no. 62, 57144-57153.

67. H.L.Y. Sin, M. Umeda, S. Shironita, A.A. Rahim and B. Saad, Aquilaria malaccensis as a green corrosion inhibitor for mild steel in $\mathrm{HCl}$ solution, Int. J. Electrochem. Sci., 2016, 11, no. 9, 7562-7575.

68. J.O. Okeniyi, C.A. Loto and A.P.I. Popoola, Total-corrosion effects of Anthocleista djalonensis and $\mathrm{Na}_{2} \mathrm{Cr}_{2} \mathrm{O}_{7}$ on steel-rebar in $\mathrm{H}_{2} \mathrm{SO}_{4}$ : Sustainable corrosion-protection prospects in microbial/industrial environment, TMS Annual Meeting, 2016, no. 1, 187192.

69. S. Chaudhary and R.K. Tak, Tribulus terrestris extracts: An eco-friendly corrosion inhibitor for mild steel in $\mathrm{H}_{2} \mathrm{SO}_{4}$ medium, Asian J. Chem., 2017, 29, no. 8, 1859-1865.

70. A. Saxena, D. Prasad and R. Haldhar, Withania somnifera extract as green inhibitor for mild steel in $8 \% \mathrm{H}_{2} \mathrm{SO}_{4}$, Asian J. Chem., 2016, 28, no. 11, 2471-2474.

71. A. Salhi, A. Bouyanzer, I.El Mounsi, H. Bendaha, I. Hamdani, E. El Ouariachi, A. Chetouani, N. Chahboun, B. Hammouti, J.M. Desjobert and J. Costa, Chemical composition, antioxidant and anticorrosive activities of Thymus Algeriensis, J. Mater. Environ. Sci., 2016, 7, no. 11, 3949-3960.

72. J. Bhawsar, P.K. Jain and P. Jain, Experimental and computational studies of Nicotiana tabacum leaves extract as green corrosion inhibitor for mild steel in acidic medium, Alexandria Eng. J., 2015, 54, no. 3, 769-775. 
73. M. Tezeghdenti, L. Dhouibi and N. Etteyeb, Corrosion Inhibition of Carbon Steel in $1 \mathrm{M}$ Sulphuric Acid Solution by Extract of Eucalyptus globulus Leaves Cultivated in Tunisia Arid Zones, J. Bio Tribo Corros., 2015, 1:16. doi: 10.1007/s40735-015-0016-X

74. P. Muthukrishnan, P. Prakash, B. Jeyaprabha and K. Shankar, Stigmasterol extracted from Ficus hispida leaves as a green inhibitor for the mild steel corrosion in $1 \mathrm{M} \mathrm{HCl}$ solution, Arabian J. Chem., 2015.

75. G. Chen, X.-Q. Hou, Q.-L. Gao, L. Zhang, J. Zhang and J.-R. Zhao, Research on Diospyros Kaki L.f leaf extracts as green and eco-friendly corrosion and oil field microorganism inhibitors, Res. Chem. Intermed., 2015, 41, no. 1, 83-92. doi: $\underline{10.1007 / \mathrm{s} 11164-013-1170-8}$

76. L. Li, W. Xu, J. Lei, J. Wang, J. He, N. Li and F. Pan, Experimental and theoretical investigations of Michelia alba leaves extract as a green highly-effective corrosion inhibitor for different steel materials in acidic solution, $R S C A d v$., 2015, 5, no. 114, 93724-93732.

77. J. Yamuna and N. Anthony, Citrus sinensis L. leaf extract as an efficient green corrosion inhibitor for mild steel in aqueous medium, Int. J. ChemTech Res., 20142015,7 , no. 1, 37-43.

78. S. Noyel Victoria, R. Prasad and R. Manivannan, Psidium guajava leaf extract as green corrosion inhibitor for mild steel in phosphoric acid, Int. J. Electrochem. Sci., 2015, 10, no. 3, 2220-2238.

79. G. Pustaj, F. Kapor and T. Borko, Olive leaf extract as a green carbon steel corrosion inhibitor in brine solution saturated with $\mathrm{CO}_{2}$, Eur. Corros. Congr., EUROCORR, 2015, 3, 1901.

80. S.A. Umoren, Z.M. Gasem and I.B. Obot, Date palm (Phoenix dactylifera) leaf extract as an eco-friendly corrosion inhibitor for carbon steel in $1 \mathrm{M}$ hydrochloric acid solution, Anti-Corros. Methods Mater., 2015, 62, no. 1, 19-28.

81. M. Mehdipour, B. Ramezanzadeh and S.Y. Arman, Electrochemical noise investigation of Aloe plant extract as green inhibitor on the corrosion of stainless steel in $1 \mathrm{M} \mathrm{H}_{2} \mathrm{SO}_{4}$, J. Ind. Eng. Chem., 2015, 21, 318-327.

82. T. Haloui, Y. Kharbach, Z. Tribak, M. El Azzouzi, A. Aouniti, B. Hammouti and A.B. Alaoui, The use of Pistacia lentiscus L. oil as green inhibitor for corrosion of mild steel in 1M Hydrochloric acid solution: Thermodynamic and adsorption investigations, Pharma Chem., 2015, 7, no. 9, 225-238.

83. N. Soltani and M. Khayatkashani, Gundelia tournefortii as a green corrosion inhibitor for mild steel in $\mathrm{HCl}$ and $\mathrm{H}_{2} \mathrm{SO}_{4}$ solutions, Int. J. Electrochem. Sci., 2015, 10, no. 1, $46-62$.

84. V. Rajeswari, D. Kesavan, M. Gopiraman, P. Viswanathamurthi, K. Poonkuzhali and T. Palvannan, Corrosion inhibition of Eleusine aegyptiaca and Croton rottleri leaf extracts on cast iron surface in $1 \mathrm{M} \mathrm{HCl}$ medium, Appl. Surf. Sci., 2014, 314, 537-545. 
85. S.A. Asipita, M. Ismail, M.Z.A. Majid, Z.A. Majid, C. Abdullah and J. Mirza, Green Bambusa Arundinacea leaves extract as a sustainable corrosion inhibitor in steel reinforced concrete, J. Cleaner Prod., 2014, 67, 139-146.

86. K. Boumhara, F. Bentiss, M. Tabyaoui, J. Costa, J.-M. Desjobert, A. Bellaouchou, A. Guenbour, B. Hammouti and S.S. Al-Deyab, Use of Artemisia Mesatlantica essential oil as Green corrosion inhibitor for mild steel in $1 \mathrm{M}$ hydrochloric acid solution, Int. J. Electrochem. Sci., 2014, 9, no. 3, 1187-1206.

87. C.A. Loto, O.O. Joseph and A.P.I. Popoola, Corrosion inhibitive behaviour of camellia sinensis on aluminium alloy in $\mathrm{H}_{2} \mathrm{SO}_{4}$, Int. J. Electrochem. Sci., 2014, 9, no. 3, $1221-$ 1231.

88. A. Sirajunnisa, M.I. Fazal Mohamed and A. Subramania, Vitex negundo leaves extract as green inhibitor for the corrosion of aluminium $1 \mathrm{~N} \mathrm{NaOH}$ solution, J. Chem. Pharm. Res., 2014, 6, no. 1, 580-588.

89. L.Y.S. Helen, A.A. Rahim, B. Saad, M.I. Saleh and P.B. Raja, Aquilaria crassna leaves extracts - A green corrosion inhibitor for mild steel in $1 \mathrm{M} \mathrm{HCl}$ medium, Int. J. Electrochem. Sci., 2014, 9, no. 2, 830-846.

90. F. Aouinti, H. Elmsellem, A. Bachiri, M.-L. Fauconnier, A. Chetouani, B. Chaouki, A. Aouniti and B. Hammouti, Plants as a source of green corrosion inhibitors on mild steel in hydrochloric acid: The case of oil extract of leaves of Pistacia lentiscus from Saidia Morocco, J. Chem. Pharm. Res., 2014, 6, no. 7, 10-23.

91. S.O. Adejo, M.M. Ekwenchi, J.U. Ahile, J.A. Gbertyo, B. Ishua and A. Akombor, Modeling of adsorption isotherm for methanol leaf extract of Manihot esculentum as green corrosion inhibitor of corrosion of mild steel in $\mathrm{HCl}$ medium, J. Corros. Sci. Eng., 2014, 17, 1-13.

92. M. Pitchaipillai, K. Raj, J. Balasubramanian and P. Periakaruppan, Benevolent behavior of Kleinia grandiflora leaf extract as a green corrosion inhibitor for mild steel in sulfuric acid solution, Int. J. Miner., Metall. Mater., 2014, 21, no. 11, 1083-1095.

93. Z.V.P. Murthy and K. Vijayaragavan, Mild steel corrosion inhibition by acid extract of leaves of Hibiscus sabdariffa as a green corrosion inhibitor and sorption behavior, Green Chem. Lett. Rev., 2014, 7, no. 3, 209-219.

94. A. Khadraoui, A. Khelifa, H. Hamitouche and R. Mehdaoui, Inhibitive effect by extract of Mentha rotundifolia leaves on the corrosion of steel in $1 \mathrm{M} \mathrm{HCl}$ solution, Res. Chem. Intermed., 2014, 40, no. 3, 961-972.

95. A. Nahlé, I. Almaidoor and I. Abdel-Rahman, UAE Rhazya Stricta Decne extract as a corrosion inhibitor for mild steel in $\mathrm{HCl}$ solution, Anti-Corros. Methods Mater., 2014, 61, no. 4, 261-266.

96. R. Suarez-Hernandez, J.G. Gonzalez-Rodriguez， G.F. Dominguez-Patiño and A. Martinez-Villafañe, Use of Opuntia ficus extract as a corrosion inhibitor for carbon steel in acidic media, Anti-Corros. Methods Mater., 2014, 61, no. 4, 224-231. 
97. A.S. Johnson, I.B. Obot and U.S. Ukpong, Green synthesis of silver nanoparticles using Artemisia annua and Sida acuta leaves extract and their antimicrobial, antioxidant and corrosion inhibition potentials, J. Mater. Environ. Sci., 2014, 5, no. 3, 899-906.

98. Y. El Ouadi, A. Bouyanzer, L. Majidi, J. Paolini, J.M. Desjobert, J. Costa, A. Chetouani and B. Hammouti, Salvia officinalis essential oil and the extract as green corrosion inhibitor of mild steel in hydrochloric acid, J. Chem. Pharm. Res., 2014, 6, no. 7, 1401-1416.

99. C.A. Loto, O.O. Joseph and R.T. Loto, Adsorption and inhibitive properties of Camellia Sinensis for aluminium alloy in $\mathrm{HCl}$, Int. J. Electrochem. Sci., 2014, 9, no. 7, 3637-3649.

100. G. Ji, S.K. Shukla, P. Dwivedi, S. Sundaram, E.E. Ebenso and R. Prakash, Parthenium hysterophorus plant extract as an efficient green corrosion inhibitor for mild steel in acidic environment, Int. J. Electrochem. Sci., 2012, 7, no. 10, 9933-9945.

101. S. Rajendran, M. Agasta, R. Bama Devi, B. Shymala Devi, K. Rajam and J. Jeyasundari, Corrosion inhibition by an aqueous extract of Henna leaves (Lawsonia inermis L), Zast. Mater., 2009, 50, no. 2, 77-84.

102. P. Nithyadevi, J. Sathiyabama, S. Rajendran, R. Joseph Rathish and S. Santhana Prabha, Corrosion resistance of mild steel in simulated concrete pore solution in presence of Green inhibitor, Int. J. Nano. Corr. Sci. Engg., 2015, 2, no. 4, 1-9.

103. V. Johnsirani, J. Sathiyabama, S. Rajendran and A. SuriyaPrabha, Inhibitory Mechanism of Carbon Steel Corrosion in SeaWater by an Aqueous Extract of Henna Leaves, Int. Scholarly Res. Network ISRN Corrosion, 2012, 1-9.

104. N. Karthiga, S. Rajendran, P. Prabhakar and S. Shanmugapriya, Unpublished results. 